\title{
Protein Kinase D Controls the Integrity of Golgi Apparatus and the Maintenance of Dendritic Arborization in Hippocampal Neurons
}

\author{
Katalin Czöndör, ${ }^{*+}$ Kornelia Ellwanger, ${ }^{+}$Yannick F. Fuchs, ${ }^{+}$Sylke Lutz, ${ }^{+}$ \\ Márton Gulyás, ${ }^{*}$ Isabelle M. Mansuy, ${ }^{\ddagger}$ Angelika Hausser, ${ }^{+}$Klaus Pfizenmaier, ${ }^{\dagger}$ \\ and Katalin Schlett*t
}

\begin{abstract}
*Department of Physiology and Neurobiology, Eötvös Loránd University, Budapest, Hungary H-1117; ${ }^{+}$Institute of Cell Biology and Immunology, University Stuttgart, D-70569 Stuttgart, Germany; and ¥Brain Research Institute, Medical Faculty of University of Zürich, CH-8057 Zürich, Switzerland, and Department of Biology of the Swiss Federal Institute of Technology, CH-8092 Zürich, Switzerland
\end{abstract}

Submitted September 19, 2008; Revised December 22, 2008; Accepted February 2, 2009

Monitoring Editor: Vivek Malhotra

\begin{abstract}
Protein kinase D (PKD) is known to participate in various cellular functions, including secretory vesicle fission from the Golgi and plasma membrane-directed transport. Here, we report on expression and function of PKD in hippocampal neurons. Expression of an enhanced green fluorescent protein (EGFP)-tagged PKD activity reporter in mouse embryonal hippocampal neurons revealed high endogenous PKD activity at the Golgi complex and in the dendrites, whereas PKD activity was excluded from the axon in parallel with axonal maturation. Expression of fluorescently tagged wild-type PKD1 and constitutively active PKD1 ${ }^{\text {5738/742E }}$ (caPKD1) in neurons revealed that both proteins were slightly enriched at the trans-Golgi network (TGN) and did not interfere with its thread-like morphology. By contrast, expression of dominant-negative kinase inactive PKD1 ${ }^{\mathrm{K} 612 \mathrm{~W}}$ (kdPKD1) led to the disruption of the neuronal Golgi complex, with kdPKD1 strongly localized to the TGN fragments. Similar findings were obtained from transgenic mice with inducible, neuron-specific expression of kdPKD1-EGFP. As a prominent consequence of kdPKD1 expression, the dendritic tree of transfected neurons was reduced, whereas caPKD1 increased dendritic arborization. Our results thus provide direct evidence that PKD activity is selectively involved in the maintenance of dendritic arborization and Golgi structure of hippocampal neurons.
\end{abstract}

\section{INTRODUCTION}

Neurons are nondividing and extremely polarized cells, with enormous membrane surface compared with the size of the soma. These features assume a highly specialized secretory machinery, which resembles in certain parts the directed transport mechanisms described in polarized nonneuronal cells (Winckler and Mellman, 1999; Horton and Ehlers, 2004). The vast neuronal membrane surface is functionally and structurally divided into axonal and somatodendritic compartments, possessing specific lipid and protein components required for the spatially different functions, e.g., for pre- or postsynaptic activity (Dresbach et al., 2001; Sheng, 2001; Bresler et al., 2004). The constitutive and precisely directed supply of surface membrane components is indispensable for the function of neurons, especially when considering that postmitotic neurons normally serve throughout the life span of the

This article was published online ahead of print in $M B C$ in Press (http:/ / www.molbiolcell.org/cgi/doi/10.1091/mbc.E08-09-0957) on February 11, 2009.

Address correspondence to: Katalin Schlett (schlettk@ludens.elte.hu).

Abbreviations used: diC8, 1,2-dioctanoylglycerol; DIV, days in vitro; Dox, doxycycline; PDBu, phorbol 12,13-dibutyrate; PI4KIII $\beta$, phosphatidylinositol 4-kinase III $\beta$; PKD, protein kinase D; TGN, trans-Golgi network; VAMP4, vesicle-associated membrane protein 4 . organism. Up to now, we are far from understanding how the extreme polarization has been evolved and is maintained in neurons.

Despite a likely central role in neuronal morphogenesis and membrane trafficking, little is known about the special structure and transport features of the neuronal Golgi apparatus. A thread-like and reticular structure of the Golgi apparatus has been already described in many neuronal types (Takamine et al., 2000; Fujita and Okamoto, 2005; Horton et al., 2005). Central neuronal Golgi complex is localized in the soma and often extends into the principal dendrites, but Golgi elements were also found as discontinuous structures in the distal dendrites, often near synaptic contacts or in dendritic spines (Gardiol et al., 1999; Pierce et al., 2001; Sytnyk et al., 2002; Horton and Ehlers, 2003). The dispersed localization of the Golgi apparatus indicates unique spatial regulation of the neuronal secretory pathway compared with other mammalian cells. Recent investigations on hippocampal pyramidal cells indicated that Golgi outposts located in the dendritic shafts (Sytnyk et al., 2002; Horton and Ehlers, 2003) and the perinuclear Golgi apparatus oriented toward the longest dendrite (Horton et al., 2005) can provide the necessary membrane supply for intensive dendritic growth. In axonal outgrowth and extension, an increasing amount of data suggests that membrane supply and targeting of proteins are regulated in a different way compared with dendritic elongation (Silverman et al., 2001; 
Horton et al., 2005; Lein et al., 2007; Takemoto-Kimura et al., 2007; Ye et al., 2007).

Based on studies in nonneuronal cells, protein kinase D (PKD) is known to play a key regulatory role in secretory pathways. The PKD family has been recently recognized as a separate family among the serine-threonine kinases and comprises three isoforms: PKD1, PKD2 and PKD3 (Van Lint et al., 2002). PKD can link several intracellular signaling cascades, indicating a role of the kinase in diverse cellular functions, including immune responses, apoptosis, or cell proliferation (Rykx et al., 2003; Wang, 2006). Moreover, in resting cells, PKD is mainly cytoplasmic, but a fraction is recruited to the Golgi apparatus where it can direct the fission of vesicles specifically destined to the cell surface (Liljedahl et al., 2001; Hausser et al., 2005). Furthermore, studies in polarized epithelial cells revealed that PKD is selectively involved in basolateral membrane protein transport (Yeaman et al., 2004). PKD was also reported to regulate lipid transfer between the endoplasmic reticulum and the Golgi apparatus (Fugmann et al., 2007).

All three PKD isoforms are expressed in the CNS already at embryonic stages in mice (Oster et al., 2006; Ellwanger et al., 2008). PKD activity has been recently implicated in regulating early neuronal polarization (Yin et al., 2008), early dendritic development (Horton et al., 2005), or selective transport and endocytosis of dendritic proteins (Bisbal et al., 2008). In the present work, we have used a newly developed reporter of PKD activity and studied transgenic mice with neuron-specific, inducible expression of a dominant-negative-acting PKD1 mutant to unravel the role of PKD in mature hippocampal neurons, both in vitro and in vivo. We show that active PKD is present at the trans-Golgi network (TGN) and in the somatodendritic compartments and that it regulates the integrity of the Golgi complex and the maintenance of the dendritic tree. Importantly, PKD activity is excluded from the axons, indicating a polarized action of PKD in the somatodendritic compartment of mature neurons.

\section{MATERIALS AND METHODS}

\section{Animals}

CD1, CaMKII $\alpha$ rtTA2 (Michalon et al., 2005), or kdPKD1-enhanced green fluorescent protein (EGFP) transgenic mice were housed in the Animal Facility of the Biological Institute, Eotvos Lorand University, or of the Institute of Cell Biology and Immunology, University of Stuttgart, at $22 \pm 1^{\circ} \mathrm{C}$, with 12 -h light/dark cycles and with ad libitum access to food and water. All procedures were performed under the supervision of Local Animal Care Committee, in agreement with the European Union and Hungarian legislation (Budapest) or approved by the Regierungspräsidium Stuttgart. All experiments were complied with local guidelines and regulations for the use of experimental animals (35-9185.81/0209, 35-9185.81/0247 and 878/003/2004 for the experiments carried out in Stuttgart or in Budapest, respectively).

\section{Generation of kdPKD1-EGFP Transgenic Mouse Line}

An EGFP-tagged version of human kinase-dead (kd) PKD1 (K612W) cDNA was inserted into the multiple cloning site of pBI-5 (Baron et al., 1995) next to the tet-operator and human cytomegalovirus (hCMV) promoter sequences. The construct contained a downstream rabbit $\beta$-globin intron/polyadenylation signal. Vector sequences were excised by digestion with NarI and BsrBI. Pronucleus microinjection was performed by standard procedures (Nagy, 2003). Tail DNA from founder mice was digested with HindIII and analyzed by Southern blotting with a 577-bp EGFP probe. The 4.7-kb fragment indicated the integration of the transgene as head-to-tail array of multiple copies into the genome, whereas the $\approx 5.8$-kb fragment denoted adjacent integration into the original genome. Polymerase chain reaction (PCR) genotyping was performed with PKD1-specific primers (forward, 5'-TTG GTC GTG AGA AGA GGT CAA ATT C-3'; reverse, 5'-CAC CAA GGC AGT TGT TTG GTA CTT T-3'), giving an amplification product of 246 base pairs for transgenic kdPKD1 and a fragment of 399 bp for endogenous PKD1. Frequency of transgenic littermates occurred in Mendelian manner.
To create a neuron-specific transgene expressing pattern, kdPKD1-EGFP mice were bred with mice carrying rtTA2 under the control of CaMKII $\alpha$ promoter (Michalon et al., 2005). Genotyping for rtTA2 was carried out as described in Michalon et al. (2005). To induce kdPKD1-EGFP expression in vivo, doxycycline hyclate (Dox; Fagron, Barsbüttel, Germany) was administrated in wet food at 6-mg/g dose. Doxycycline-containing food was prepared daily, and pellets were covered to protect from light. Ten- to 14-wk-old animals received doxycycline-containing food for $8 \mathrm{~d}$ to $4 \mathrm{wk}$.

\section{Expression Constructs}

Fluorescently tagged human wtPKD1-GFP, kdPKD1-GFP (PKC $\mu^{\mathrm{K} 612 \mathrm{~W}}$-EGFP), PKD1 1 PH-GFP, or caPKD1-GFP (PKD1 ${ }^{5738 / 742 E}$ ) plasmids have been described previously (Hausser et al., 1999, 2002, 2005). pEGFP-C1, pEGFP-N1, and pmCherry-N1 vectors were from Clontech Europe (Saint-Germain-enLaye, France) and A. Jeromin, Allen Institute for Brain Science (Seattle, WA), respectively. The PKD activity reporter (PKDrep) was generated by annealing oligonucleotides containing the cDNA sequence for the linkers and phosphorylation substrate region of phosphatidylinositol 4-kinase III $\beta$ (PI4KIII $\beta$ ) (forward, $5^{\prime}$-a gct tta ggt ggt tct ggt ggt agc agc aac ctg aaa cga aca gcc agc aac cct aaa gtg gag aat gag ggt ggt tct ggt ggt g- $3^{\prime}$ and reverse, $5^{\prime}$-tc gac acc acc aga acc acc ctc att ctc cac ttt agg gtt get gge tgt tcg ttt cag gtt get get acc acc aga acc acc taa- $\left.-3^{\prime}\right)$. HindIII and SalI sites were incorporated at the $5^{\prime}$ and $3^{\prime}$ ends of the oligonucleotides, respectively. The mutation S294A was incorporated by exchanging the triplet $a g c$ for $g c c$ within the oligonucleotides. Annealed oligonucleotides were inserted in the multicloning site of the pEGFP-C1 vector (Clontech Europe) by using the HindIII and Sall restriction sites. Generation and characterization of Golgi targeted PKD activity reporter constructs are described in detail elsewhere (Fuchs et al., 2009).

\section{Cell Cultures}

Primary cultures of embryonal hippocampal cells were prepared from CD1 mice on embryonic day 18. After aseptically removing hippocampus from the skull, tissue was freed from meninges and incubated in $0.05 \%$ trypsin-EDTA (Invitrogen, Csertex, Hungary) solution with $0.05 \%$ DNaseI in phosphatebuffered saline (PBS) for $15 \mathrm{~min}$ at $37^{\circ} \mathrm{C}$. After a brief centrifugation, cells were triturated in Neurobasal (NB) medium supplemented with B27 (Invitrogen) containing $5 \%$ fetal calf serum (FCS; Sigma, Budapest, Hungary), $0.5 \mathrm{mM}$ GlutaMAX (Invitrogen), $40 \mu \mathrm{g} / \mathrm{ml}$ gentamicin (Hungaropharma, Budapest, Hungary), and $2.5 \mu \mathrm{g} / \mathrm{ml}$ amphotericin B and filtered through a sterile polyester mesh with $42-\mu \mathrm{m}$ pore size (EmTek, Budapest, Hungary). Cell number was determined by trypan blue exclusion, and cells were seeded in NB culture medium onto poly-L-lysine (Sigma) $-1 \mu \mathrm{g} / \mathrm{cm}^{2}$ laminin (Sigma)coated glass coverslips (13 mm in diameter) in 24 -well plates at $6 \times 10^{4}$ cells $/ \mathrm{cm}^{2}$ cell density for microscopic observations. For Western blot analyses, cells were seeded into 12 -well plates at $4 \times 10^{5}$ cells / well cell density; for live cell imaging experiments, $4 \times 10^{5}$ cells were plated onto poly-L-lysinelaminin coated glass-bottomed 35-mm Petri dishes (Greiner, Frickenhausen, Germany). Cytosin-arabinofuranoside (CAR, $10 \mu \mathrm{M}$; Sigma) was added to the cultures $48 \mathrm{~h}$ after plating, and then one third of the culture medium was changed to Neurobasal medium supplemented with B27 without FCS on the fourth day of cultivation. One third of the medium was replaced every 3-4 d thereafter. Cells were cultivated for $10 \mathrm{~d}$ at $37^{\circ} \mathrm{C}$ in $5 \% \mathrm{CO}_{2}, 95 \%$ air atmosphere. Immunocytochemical stainings revealed relatively low amount $(<5 \%)$ of interneurons containing GABA and a high level of vesicular glutamate transporter-1 (vGluT1) in our cultures (data not shown).

Nine days after plating, transfection of hippocampal cells was carried out using Lipofectamine 2000 (Invitrogen) according to the manufacturer's instructions. In 24-well plates, $0.3-0.5 \mu \mathrm{g}$ of plasmid DNA was mixed with Lipofectamine in a $1 \mu \mathrm{g}: 2 \mu \mathrm{l}$ ratio. Medium was changed 5-8 h after the transfection to the original cultivation medium, and cells were analyzed at the indicated time points after transfection. Phorbol ester treatment of cultures was carried out either by phorbol 12,13-dibutyrate (PDBu; $1 \mu \mathrm{M} ; 2 \mathrm{mM}$ stock solution dissolved in dimethyl sulfoxide [DMSO]; Sigma Aldrich Chemie Gmbh, Munich, Germany) or by the cell-permeable DAG analogue sn-1,2-dioctanoylglycerol (diC8; $25 \mu \mathrm{M} ; 25 \mathrm{mM}$ stock solution dissolved in DMSO; Sigma Aldrich Chemie Gmbh) treatment in the culture medium.

Human embryonic kidney (HEK) 293T cells were maintained in RPMI 1640 medium supplemented with $10 \%$ fetal calf serum (all from Invitrogen, Karlsruhe, Germany). For transient transfections of the indicated plasmids, HEK293T cells were transfected with TransIT293 (Mirus Bio, Madison, WI) according to the manufacturers instructions.

\section{Immunostaining}

Hippocampal cultures were fixed on the 10th day after plating (days in vitro [DIV] 10) for $20 \mathrm{~min}$ with cold $4 \%$ paraformaldehyde, $4 \%$ sucrose in PBS. After rinsing, cells were permeabilized with $0.1 \%$ Triton X-100 for $5 \mathrm{~min}$. Nonspecific antibody binding was blocked by incubation with $2 \%$ bovine serum albumin (BSA), $0.1 \%$ Na-azide in PBS (PBS-BSA) for $1 \mathrm{~h}$ at room temperature. Primary antibodies such as anti-GM130 (mouse immunoglobulin G [IgG]1, 1:250; BD Biosciences Transduction Laboratories, Lexington, KY) anti-vesicle-associated membrane protein (VAMP) 4 (rabbit, 1:300; Sigma), antipSer294 (rabbit, 1:750; Hausser et al., 2005), anti-FLAG (mouse, 1:500; Sigma), 
or anti-PKD1 (JP2; monoclonal IgG, 1:500; Johannes et al., 1995) were diluted in PBS-BSA and used at $4^{\circ} \mathrm{C}$ overnight. Anti-mouse IgGs conjugated with Alexa 633 (1:500; Invitrogen) or anti-rabbit-Alexa 546 (1:1000; Invitrogen) or $\mathrm{F}_{(\mathrm{ab}) 2}$ fragments of anti-mouse IgG conjugated with Alexa 488 (for visualizing JP2 signal, 1:500; Invitrogen) were used for $1.5 \mathrm{~h}$ at room temperature. Cultures were mounted with Mowiol 4.88 (Polysciences, Eppelheim, Germany) supplemented with bis-benzimide.

Wild-type (wt) or double transgenic mice were deeply anesthetized with isoflurane and were transcardially perfused with $4 \%$ paraformaldehyde (Taab, Aldermaston, United Kingdom, wt/vol in PBS). Brains were postfixed overnight and cryoprotected in $30 \%$ sucrose. Then, $30-\mu \mathrm{m}$ coronal sections from the area between bregma -3 to $-1.5 \mathrm{~mm}$ were incubated with $0.5 \%$ Triton X-100-PBS for 30 min and nonspecific antibody binding was blocked by incubating the sections with $2 \%$ BSA, $0.3 \%$ Triton X-100, $0.1 \%$ Na-azide in PBS (blocking solution) for $1 \mathrm{~h}$ at room temperature. Anti-VAMP4 diluted in blocking solution (rabbit, 1:300; Sigma) was applied for $1 \mathrm{~d}$ at $4^{\circ} \mathrm{C}$. After washing with PBS $(4 \times 15 \mathrm{~min})$, sections were incubated with anti-rabbit IgGs conjugated with Alexa 546 (1:300; Invitrogen) in blocking solution for $1.5 \mathrm{~h}$ at room temperature. Sections were mounted onto poly-L-lysine-coated slides and covered with Mowiol supplemented with bis-benzimide. In all cases, control samples were processed similarly, except that the first layer antibodies were omitted.

\section{Microscopy}

Microscopic analyses were carried out only from those transfected neurons, which had a clear distinction between dendrites and the axon, a complete dendritic tree with dendritic spines and an intact nucleus. Pictures were taken with an HS CellObserver inverse microscope (Carl Zeiss, Jena, Germany) equipped with AxioCam HR 12 bit camera, the Apotome system, and with Plan Neofluar $10 \times / 0.3$ M27, Plan Apochromat $20 \times / 0.8$ M27 or Plan Apochromat $63 \times / 1.4$ oil differential interference contrast (DIC) M27 objective lenses. 3D reconstructions of the Golgi structure were made out of 28-33 $\mathrm{z}$-stacks by using the Inside $4 \mathrm{D}$ tool of the AxioVision 4.6 software. In this case, adjacent $\mathrm{z}$-stacks were recorded by $0.15-\mu \mathrm{m}$ steps. Confocal microscopy was performed with a TCS SP2 confocal scanning microscope (Leica, Wetzlar Germany) at Airy pinhole using 488-, 543-, and 633-nm excitation and a $100 \times / 1.4$ HCX PL APO objective lens. Wide-field fluorescent pictures were taken by a BX51 microscope (Olympus, Tokyo, Japan) equipped with a FluoViewII camera and the AnalysisPro software, by using either $60 \times / 1.4$ or $100 \times / 1.2$ oil Plan Apochromat phase contrast objectives.

Live cell imaging observations were carried out with the HS CellObserver and with a 488-nm blue led illumination by the Colibri system. Neurons were observed by the Plan Apochromat $63 \times / 1.4$ oil DIC M27 objective lens and with the AxioCam HR camera with 200-ms frame rate and $2 \times 2$ binning. During imaging, cultures were kept in imaging buffer $(142 \mathrm{mM} \mathrm{NaCl}, 5.4 \mathrm{mM}$ $\mathrm{KCl}, 1.8 \mathrm{mM} \mathrm{CaCl}_{2}, 1 \mathrm{mM} \mathrm{NaH}_{2} \mathrm{PO}_{4}, 25 \mathrm{mM}$ HEPES, $5 \mathrm{mM}$ glucose, and 0.8 $\mathrm{mM} \mathrm{MgCl}$, $\mathrm{pH} \mathrm{7.4)}$ and at $37^{\circ} \mathrm{C}$. In the PDBu treatment, $1 \mu \mathrm{M}$ PDBu was applied to the observed Petri dish directly on the stage of the microscope.

\section{Quantitative Analyses of Microscopy Data}

Golgi apparatus enrichment index (GI) was calculated to quantify the intracellular distribution of EGFP-tagged PKD1 constructs in DIV10 neurons. The 12-bit fluorescent images were recorded below saturation level with Plan Apochromat $63 \times / 1.4$ oil DIC M27 objective lens, by using the Apotome system (Carl Zeiss). Average fluorescent intensity was calculated from $5 \times 1$ $\mu \mathrm{m}^{2}$ areas located over the cytoplasm or over the VAMP4-stained TGN structures followed by subtracting background fluorescent intensity. Average TGN intensity values were compared with the corresponding cytoplasmic intensity values, resulting in GI for each cell. Average GI was calculated from 13 EGFP, 13 wtPKD1-EGFP, 26 kdPKD1-EGFP and 20 caPKD1-EGFP transfected neurons.

Golgi morphology was analyzed in three independent cultures, transfected with the indicated constructs in parallel. In total, Apotome recordings were made from 34 EGFP-, 46 wtPKD1-EGFP-, 37 kdPKD1-EGFP-, and 53 caPKD1EGFP-transfected neurons, by using the Plan Apochromat $63 \times / 1.4$ oil DIC M27 objective lens. Only those cells were taken into account where VAMP4 immunostaining of the Golgi complex was clearly detectable. Neuronal Golgi morphology was regarded as thread-like when cis- and trans-Golgi structures occurred as reticular networks with mostly continuous filaments. Dispersed Golgi structure had much shorter (if any) reticular structures and many small fragments, all labeled with VAMP4 and GM130 as well. Pictures were analyzed by a person unaware of the constructs used for the transfection.

Dendritic tree morphology of 27 EGFP-, 23 PKD1-EGFP-, 28 kdPKD1-EGFP-, and 27 caPKD1-EGFP-transfected cells was analyzed by a modified Sholl analysis. A template consisting of circles with consecutively increasing diameter (with $40-\mu \mathrm{m}$ steps) was placed on the inverted pictures of the transfected neurons, and the number of intersections was determined at every level.

Changes in the average expression levels of kdPKD1-EGFP-transfected neurons were analyzed using 8-bit wide-field fluorescent images recorded by the BX51 Olympus microscope with identical exposure and gamma settings. Average intensity values were calculated from three parallel cultures by determining average fluorescent intensity in regions of interest drawn manually around the soma of the transfected neurons.

During quantitative evaluation of the microscopy data, only cells with intact nuclei (visualized by 4,6-diamidino-2-phenylindole [DAPI] staining) were taken for analyses. For statistical evaluation, Student's $t$ test was used in all cases $(p<0.05)$.

\section{Western Blot}

Whole cell extracts from transfected HEK293T cells and from neuronal cultures were obtained by harvesting in lysis buffer (1\% Triton X-100, $20 \mathrm{mM}$ Tris, pH 7.5, $150 \mathrm{mM} \mathrm{NaCl}, 1 \mathrm{mM}$ EGTA, $1 \mathrm{mM}$ EDTA, $1 \mathrm{mM}$ sodium orthovanadate, $10 \mathrm{mM}$ sodium fluoride, $20 \mathrm{mM} \beta$-glycerophosphate, and Complete protease inhibitors [Roche Diagnostics, Mannheim, Germany]) Brain lysates were prepared by homogenization in $5 \mu \mathrm{l}$ of lysis buffer per milligram of tissue. Lysates were clarified by centrifugation at $10,000 \times g$ for $10 \mathrm{~min}$. Protein content of the supernatant was determined by the Bradford reagent (Bio-Rad, Hamburg, Germany). Equal protein amount of the corresponding samples were subjected to SDS-polyacrylamide gel electrophoresis and blotted onto nitrocellulose membranes (Pall, East Hills, NY). After blocking with $0.5 \%$ blocking reagent (Roche Diagnostics) in PBS containing $0.1 \%$ Tween 20, filters were probed with specific antibodies as follows: anti-PKD1 (C20 rabbit, 1:2000; Santa Cruz Biotechnology, Santa Cruz, CA), anti-pS910 (rabbit, 1:2000; Hausser et al., 2005); anti-III $\beta$ tubulin (mouse, 1:5000; Exbio, Prague, Czech Republic), and anti-pS738/pS742 (rabbit, 1:100; Hausser et al., 2002). Proteins were visualized with horseradish peroxidase-coupled secondary antibodies (Dianova, Hamburg, Germany) by using the enhance chemiluminescence system (Pierce Chemical, Rockford, IL). Stripping of membranes was performed in $62.5 \mathrm{mM}$ Tris, $\mathrm{pH} 6.8,2 \%$ SDS, and $100 \mathrm{mM}$ $\beta$-mercaptoethanol for $30 \mathrm{~min}$ at $55^{\circ} \mathrm{C}$. Membranes were then reprobed with the indicated antibodies.

\section{RESULTS}

\section{PKD Is Localized at the Trans-Golgi Network in Neurons}

We have visualized neuronal Golgi apparatus in cultured mouse hippocampal neurons using GM130 and VAMP4 immunostaining (Figure 1). GM130 has been widely used both in nonneuronal and neuronal cells to label cis-Golgi (Nakamura et al., 1995; Horton et al., 2005), whereas VAMP4, a member of the soluble $N$-ethyl maleimide-sensitive factor adaptor receptor complex, is known to be highly enriched at the TGN and to regulate the traffic and sorting of recycling endosomes (Steegmaier et al., 1999; Tran et al., 2007). Both GM130 and VAMP4 highlighted filamentous, perinuclear staining in cultivated neurons. The cis- and trans-Golgi compartments were located side by side, forming a reticular structure in the soma. Filamentous Golgi structures often entered one or two dendrites as well (see Supplemental Figure 1 and Supplemental Movie 1 for three-dimensional [3D] reconstruction).

In epithelial cells, PKD regulates transport processes and vesicle fission via binding to TGN membranes (Maeda et al., 2001; Diaz Anel and Malhotra, 2005; Ghanekar and Lowe, 2005). The intracellular localization of PKD was followed by introducing EGFP-tagged human wild-type PKD1 (wtPKD1-EGFP) into hippocampal neurons already possessing elaborate axonal arborization and well developed dendrites (9 d after plating; DIV9). Cultures were fixed 22-24 h after transfection and were processed for GM130 and VAMP4 immunocytochemistry (Figure 1A). Besides a rather homogenous cytoplasmic distribution, wtPKD1-EGFP was significantly enriched at the Golgi apparatus (Table 1). On closer examination, wtPKD1-EGFP was localized side by side with GM130 but showed partially overlapping localization with VAMP4-stained structures (Figure 1A, enlargements).

Phorbol ester treatment is known to cause plasma membrane translocation and activation of PKD (Zugaza et al., 1996; Matthews et al., 1999; Rey et al., 2004). In live cell imaging of hippocampal neurons, $1 \mu \mathrm{M}$ PDBu treatment led to a rapid recruitment of wtPKD1-EGFP to the plasma membrane, followed by the intracellular accumulation of the fluorescently tagged protein (Figure 1B). Immunocytochemical staining with VAMP4 confirmed that 15 min of PDBu 
A
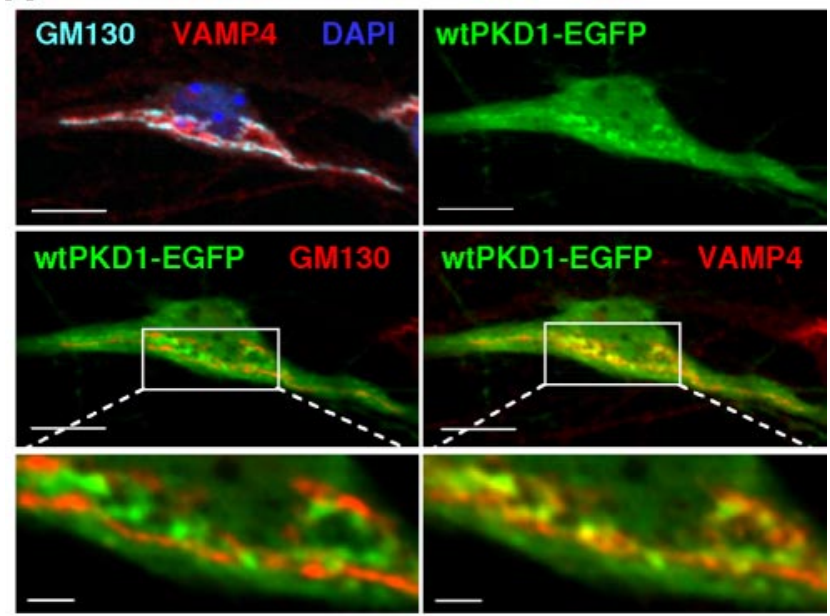

B
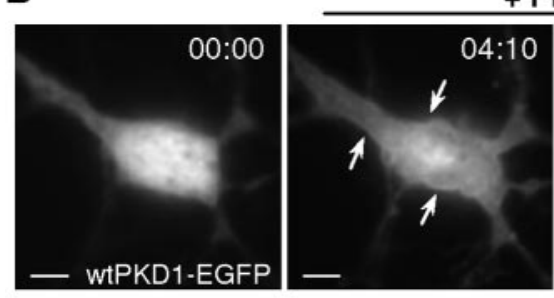

+ PDBu
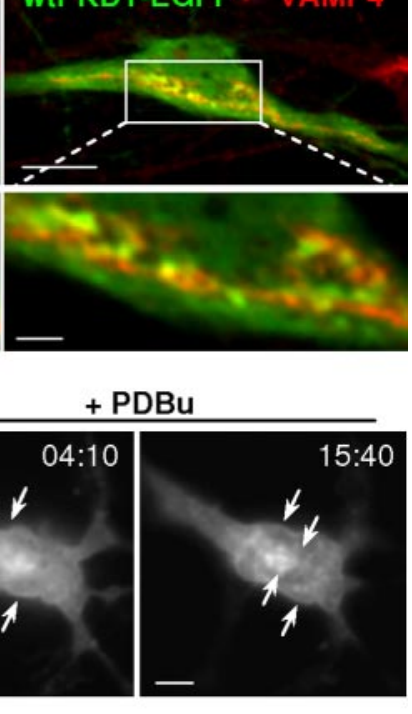

C $+\mathrm{PDBu}$
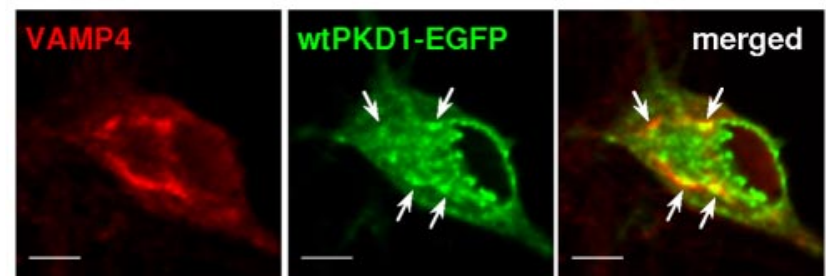

Figure 1. Localization of wtPKD-EGFP in transfected hippocampal neurons. (A) Single z-stacks from a neuron transfected by wtPKD1-EGFP and immunostained for GM130 and VAMP4. Indicated perinuclear regions are enlarged on the bottom pictures where for the sake of better comparison, GM130 and VAMP4 are similarly displayed in red color. wtPKD1-EGFP showed side-by-side localization with GM130 (left) but strongly colocalized with VAMP4stained structures (right). (B) Live cell imaging recording from a hippocampal neuron transfected with wtPKD1-EGFP and treated with $1 \mu \mathrm{M}$ PDBu. Elapsed time is indicated as minutes:seconds. PDBu treatment induced the recruitment of wtPKD1-EGFP to the plasma membrane and to internal structures (arrows). (C) Single z-stacks from a transfected neuron treated with $1 \mu \mathrm{M}$ PDBu for 15 min and immunostained with anti-VAMP4. Localization of wtPKD1-EGFP to TGN is indicated by the arrows. Bars, $10 \mu \mathrm{m}$ (top pictures in A), $2 \mu \mathrm{m}$ (enlarged regions in A), and $5 \mu \mathrm{m}$ (B and C).

treatment enhanced the translocation of wtPKD1-EGFP to the TGN as well as to the nuclear membrane and to other intracellular structures (Figure 1C). These results indicate that fluorescently labeled PKD1 behaved similarly in transfected neurons as endogenous PKD in nonneuronal cells.

\section{Endogenous PKD Is Active at the Neuronal Golgi Complex}

The consensus PKD target site has been mapped (Nishikawa et al., 1997), and antibodies raised against the phosphorylated serine of the consensus PKD target site have been successfully used to demonstrate PKD-mediated phosphorylation of various substrate molecules (Doppler et al., 2005; Hausser et al., 2005). PI4KIII $\beta$ is a known target of PKD and gets selectively phosphorylated by PKD on Ser294 (Hausser et al., 2005). To visualize endogenous PKD activity at the Golgi complex, a Golgi-targeted EGFP-tagged PKD activity reporter construct (G-PKDrep) was created using the PKDspecific substrate sequence of PI4KIII $\beta$ fused to the GRIP domain of p230, a known trans-Golgi localized protein (Fuchs et al., 2009). PKD-mediated phosphorylation of the transfected construct can be detected by an antibody specific for phosphorylated Ser294 (anti-pS294; Hausser et al., 2005). Specificity of the immunostaining was proven by transfecting a construct containing a serine to alanine mutation inside the PKD target sequence (G-PKDrep S/A).

The GRIP domain of p230 led to a selective enrichment of both EGFP-tagged constructs at the Golgi complex, the latter visualized by GM130 staining (Figure 2). As expected, pS294 antibody staining was detected only in those cells that were transfected with the PKD reporter containing the original serine site. GM130 immunostaining revealed a side-by-side localization with the pS294 signal, in accordance with the TGN-directed localization of the PKD activity reporter. Importantly, Golgi-targeted reporter was highly phosphorylated at the TGN in transfected neurons, even without phorbol ester treatment.

\section{Nontargeted PKD Activity Reporter Is a Suitable Tool to Track Endogenous PKD Activity}

The nontargeted versions of PKD activity reporter containing either serine or alanine within the PKD target site (PKDrep or PKDrep S/A, respectively) were also created (Figure 3A). Similarly to the Golgi-targeted PKD activity reporter, described in detail by Fuchs et al. (2009), PKDrep was shown to be a sensitive and specific tool to follow dynamics of PKD activation. On the cotransfection of HEK293T cells with the reporter and the indicated PKD1 constructs, mutant PKD1 lacking the inhibitory PH domain led to increased phosphorylation of the PKD target site compared with wtPKD1, whereas alanine mutation of PKDrep abolished pS294 reactivity (Figure 3B). Rapid and reversible phosphorylation of PKDrep was shown after FCS treatment of serumstarved HEK293T cells (Figure 3C). Application of diC8 (25 $\mu \mathrm{M})$, a cell-permeable DAG analogue, induced rapid changes in the localization and intensity of pS294 signal in hippocampal neurons as well (Figure 3, D-F). Thus, alike a natural substrate, phosphorylation of the reporter allows the dynamic monitoring of endogenous PKD activity.

\section{PKD Is Highly Active in Nonpolarized Neurons and in the} Somatodendritic Compartments of Mature Neurons

Endogenous activity of mouse PKD1 was detected by an antibody recognizing autophosphorylated human PKD1 (pS910; Haworth and Avkiran, 2001; Hausser et al., 2002). This result was confirmed by the increased signal intensity upon phorbol ester stimulus as well as by reprobing with a PKD1-specific antibody (Figure 4, A and C). The relative amount of PKD1 remained similar during the first $4 \mathrm{~d}$ of neuronal development in the hippocampal cultures, but it decreased slightly after the second week of plating. In contrast, the relative amount of activated PKD1 decreased rapidly in our mouse hippocampal cultures, to a much higher extent than the decline observed in total PKD1 levels (Figure 4B). These results confirm that endogenous PKD1 is present in cultured hippocampal neurons and is highly active during initial neurite elongation. 
Table 1. PKD1 constructs are enriched at the TGN of the transfected neurons

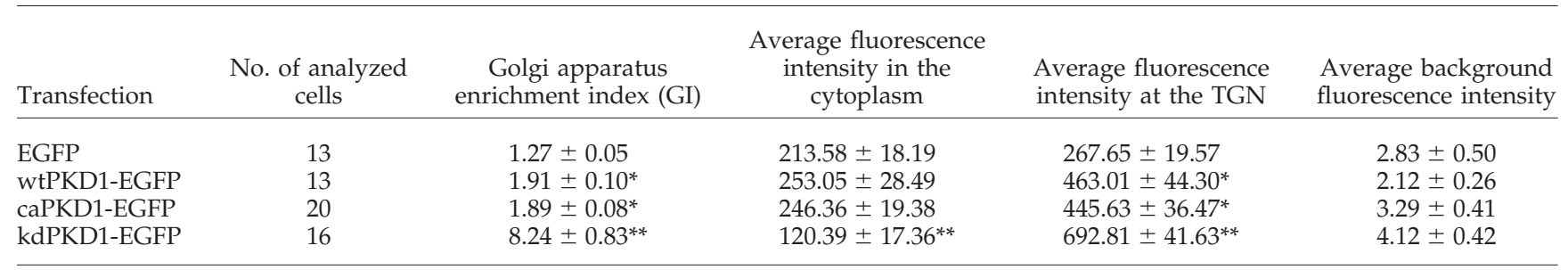

Background intensity values, average fluorescence intensity over the cytoplasmic region or over the VAMP4-positive TGN were calculated as described in Materials and Methods, at $16 \mathrm{~h}$ after transfection, by using single z-stacks from 12-bit Apotome images. Exposure settings for the individual cells were made to avoid saturation of the pixels. Because GI was calculated for each cell individually, relative increase between average Golgi complex or cytoplasmic intensity values reflects relative enrichment at the Golgi apparatus. In comparison with values obtained from EGFP-transfected neurons, wtPKD1-EGFP and caPKD1-EGFP were significantly enriched at the TGN, whereas the kdPKD1EGFP signal was reduced in the cytoplasm but highly trapped at the TGN. Data are given as mean \pm SEM. Asterisks indicate significant changes compared with the corresponding EGFP values $\left({ }^{*} \mathrm{p}<0.05 ;{ }^{* *} \mathrm{p}<0.001\right)$.

Developmental changes in the intracellular activity of endogenous PKD were also investigated using the nontargeted version of PKD activity reporters (PKDrep or PKDrep S/A; Figure 4, D-H). Both the control, alanine containing construct (PKDrep S/A; data not shown) and PKDrep were evenly distributed in the cytoplasm of the transfected neurons. In nonpolarized stage 2 neurons, PKDrep phosphorylation was evident in all neurites and in the soma (Figure 4D). In contrast, phosphorylation of PKDrep was gradually diminished in parallel with axonal maturation: stage 3 neurons with already elongated axons possessed endogenous PKD activity only in the proximal axon (Figure 4E, asterisk). In mature neurons, pS294 signal was excluded from the axons, both from the axonal initial segment (Figure 4F) or from the thinner axon collaterals (Figure 4G, arrowheads). At the same time, endogenous PKD activity was evident in the soma and in the main dendrites (Figure 4F) as well as in the smaller dendritic branches and dendritic spine heads (Figure 4G, small arrows). When neurons were cotransfected with PKDrep and the constitutive active mutant PKD1 (caPKD1-mCherry), phosphorylation of PKDrep was detected in axons coexpressing caPKD1 (Figure $4 \mathrm{H}$, arrowheads). Additionally, axonal pS294 signal was not increased even after $15 \mathrm{~min}$ of diC8 treatment (Figure $3 \mathrm{~F}$ ).
Using a monoclonal PKD1 antibody for immunostaining, PKD1 signal was clearly detected in the axons (Supplemental Figure 2). Specificity of the immunostaining was strengthened by increased signal intensity upon overexpressing wtPKD1mCherry in the axon (Supplemental Figure 2C). Therefore, the lack of pS294 staining in the axons is not due to lack of endogenous PKD protein but rather to the selective lack of endogenous PKD activity in mature axons. This indicates that PKD has a polarized activity in the somatodendritic compartment of cultured hippocampal neurons.

We also tested the consequences of overexpressing wtPKD2-mCherry or wtPKD3-FLAG on axonal PKDrep phosphorylation (Supplemental Figure 3, A and B, respectively). In both cases, pS294 signal was clearly detected in the axons of the cotransfected neurons. Additionally, interaction between the different isoforms was proved by showing that kinase inactive PKD1 $1^{\mathrm{K} 612 \mathrm{~W}}$ acts in a dominant-negative way and inhibits the activation loop (S738/S742) phosphorylation of both PKD1 and PKD2 (Supplemental Figure 3C).

\section{Expression of Dominant-Negative Kinase Inactive PKD1 Leads to the Disruption of the Golgi Complex}

In nonneuronal cells, disruption of the kinase activity of PKD leads to impaired secretory vesicle fission and tubula-
A
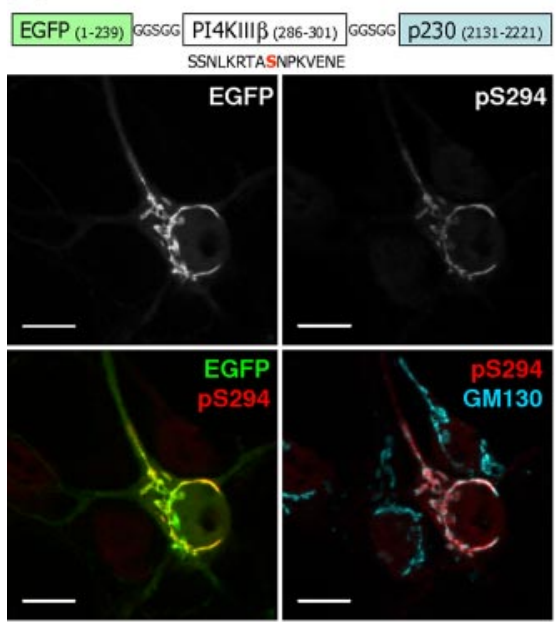

G-PKDrep
B

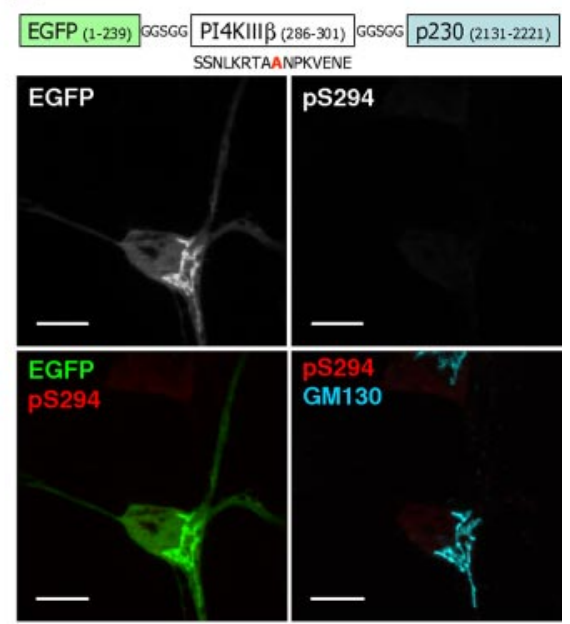

G-PKDrep S/A
Figure 2. Endogenous PKD activity is detected at the neuronal Golgi complex. Cultivated hippocampal neurons were transfected by the Golgi-targeted PKD activity reporter constructs containing the PKD-specific substrate sequence of PI4KIII $\beta$ around Ser294 (A; G-PKDrep). Golgi targeting was achieved by fusing the GRIP domain of p230 to the target sequence. In the control construct, S294 was mutated to alanine (B; G-PKDrep S/A). PKDmediated phosphorylation of the reporter was detected by using an antibody specific to the phosphorylated state of Ser294 (pS294), whereas cis-Golgi was visualized by GM130 immunostaining. Bars, $10 \mu \mathrm{m}$. 

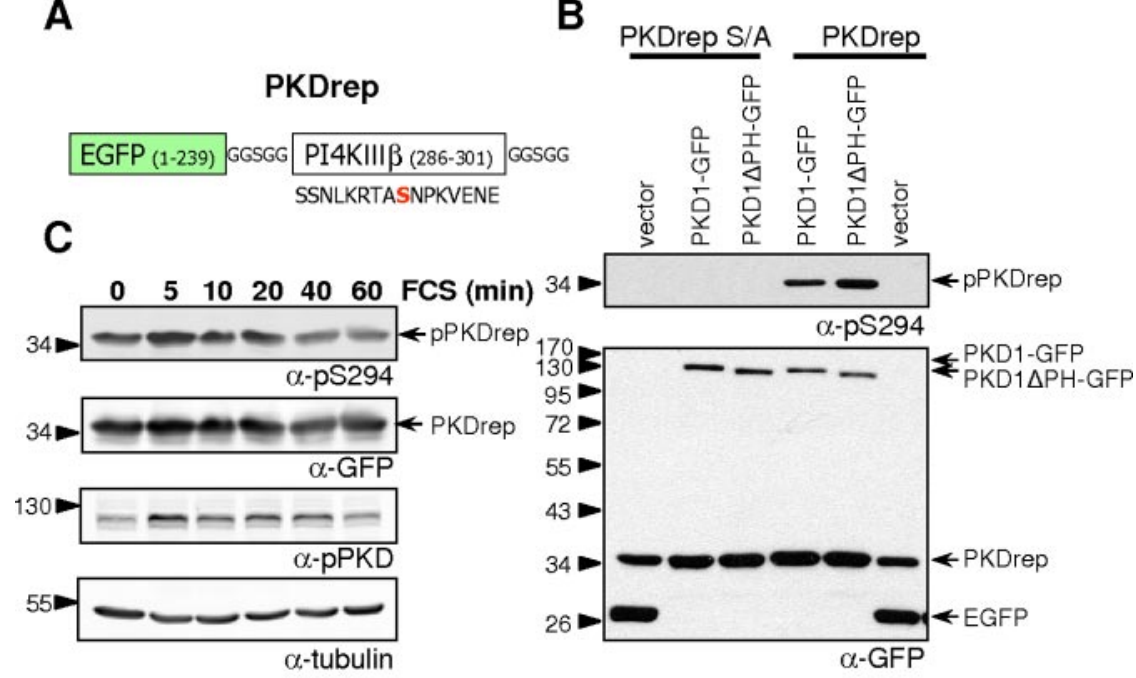

Figure 3. PKDrep is a sensitive, specific, and reversible tool to measure endogenous PKD activity. (A) Outline of the nontargeted PKDrep construct. (B) HEK293T cells were transfected with PKDrep and the S/A variant, respectively, along with an empty vector or the indicated PKD1 expression plasmids. Cells were lysed, and reporter phosphorylation was assessed by immunoblotting with the pS294specific antibody. Expression of the PKDrep and PKD1-GFP fusion proteins was verified with a GFP-specific antibody. (C) HEK293T cells transiently expressing PKDrep were serum starved overnight followed by stimulation with $10 \%$ serum for the indicated time. Phosphorylation and expression of the PKD reporter and phosphorylation of PKD on S910 were analyzed as described in B. (D-F) diC8 $(25 \mu \mathrm{M})$ treatment rapidly induced PKDrep phosphorylation in DIV10 hippocampal neurons. Strong, plasma membrane localized pS294 staining was detected already upon 2 min of diC8 treatment (E). By 15 min of diC8 treatment, elevated pS294 signal was in intracellular compartments as well $(\mathrm{F})$. Images are single $\mathrm{z}$-stacks recorded by the Apotome system under identical illumination and exposure settings and with similar processing. Axons are indicated by arrowheads on $\mathrm{E}$ and $\mathrm{F}$ (on D, the axon is out of focus). Note that axonal pS294 signal is hardly detectable even after 15 min of diC8 treatment (F). Bars, $10 \mu \mathrm{m}$.
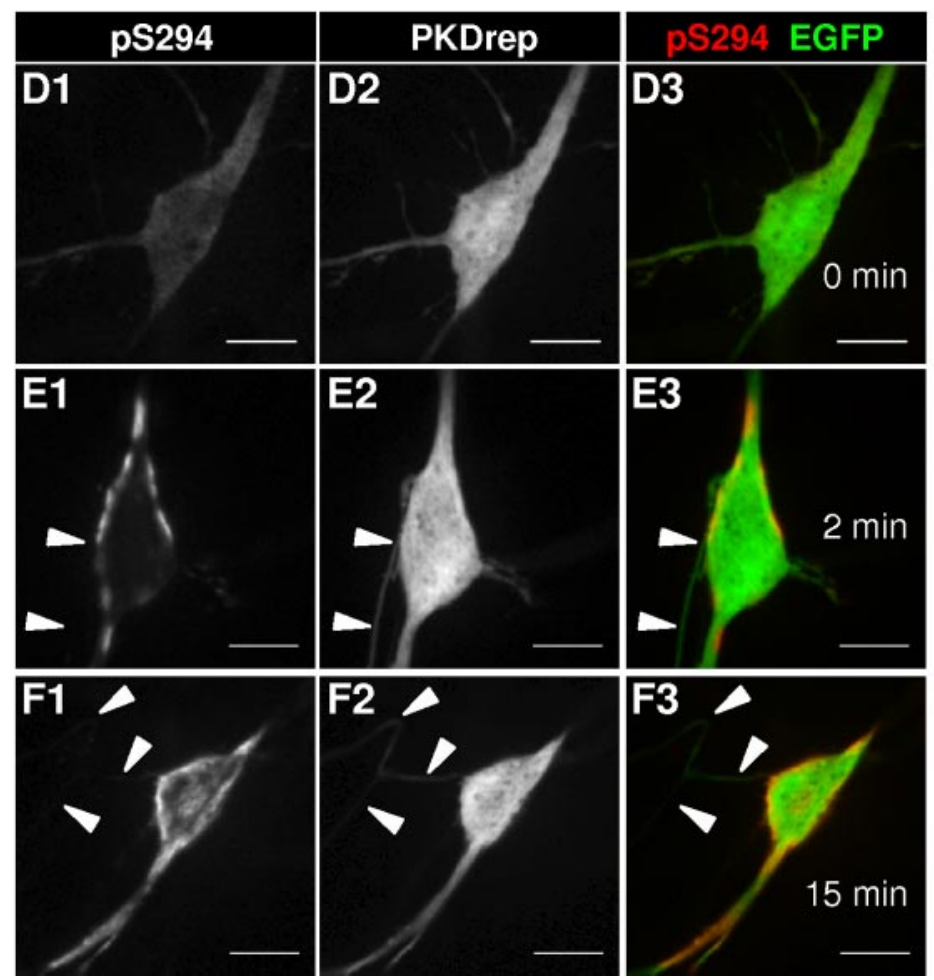

tion of the TGN (Liljedahl et al., 2001). To investigate the effects of different PKD mutations on neuronal Golgi complex morphology, DIV9 hippocampal neurons were transfected with EGFP-tagged wild-type, constitutive active and dominant-negative kinase inactive (kdPKD1-EGFP) forms of human PKD1 or the EGFP vector. Twenty-four hours after transfection, caPKD1-EGFP showed similar distribution pattern to wtPKD1-EGFP (Figure 5A vs. Figure 1A), being evenly distributed in the cytoplasm but significantly enriched around the neuronal Golgi complex (Table 1). Expression of caPKD1-EGFP also did not disturb the thread-like structure of the neuronal Golgi complex (Figure 5A and Supplemental Movie 2).

Transfection with the kinase inactive PKD1 mutant, in contrast, led to evident changes in neuronal Golgi morphology (Figure 5, B and C). VAMP4 and GM130 immunostain- ing revealed that Golgi complex of the transfected neurons was disrupted into several small fragments containing both cis- and trans-Golgi elements (Figure 5C). Besides some weak cytoplasmic distribution, kdPKD1-EGFP was strongly localized to these fragments. In many cases, a sandwich-like arrangement between GM130- and VAMP4-positive structures and kdPKD1-EGFP was observed, with VAMP4-labeled trans-Golgi parts being in the middle (Figure 5C). 3D reconstruction further supported that kdPKD1-EGFP was trapped mostly at the trans-Golgi regions (see Supplemental Movie 3).

Quantitative analysis of Golgi morphology from three independent cultures additionally confirmed the abovementioned findings by showing that the ratio of transfected neurons possessing dispersed Golgi complex was significantly increased upon kdPKD1-EGFP expression but did not 
A

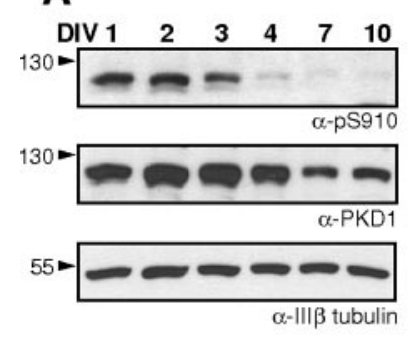

B
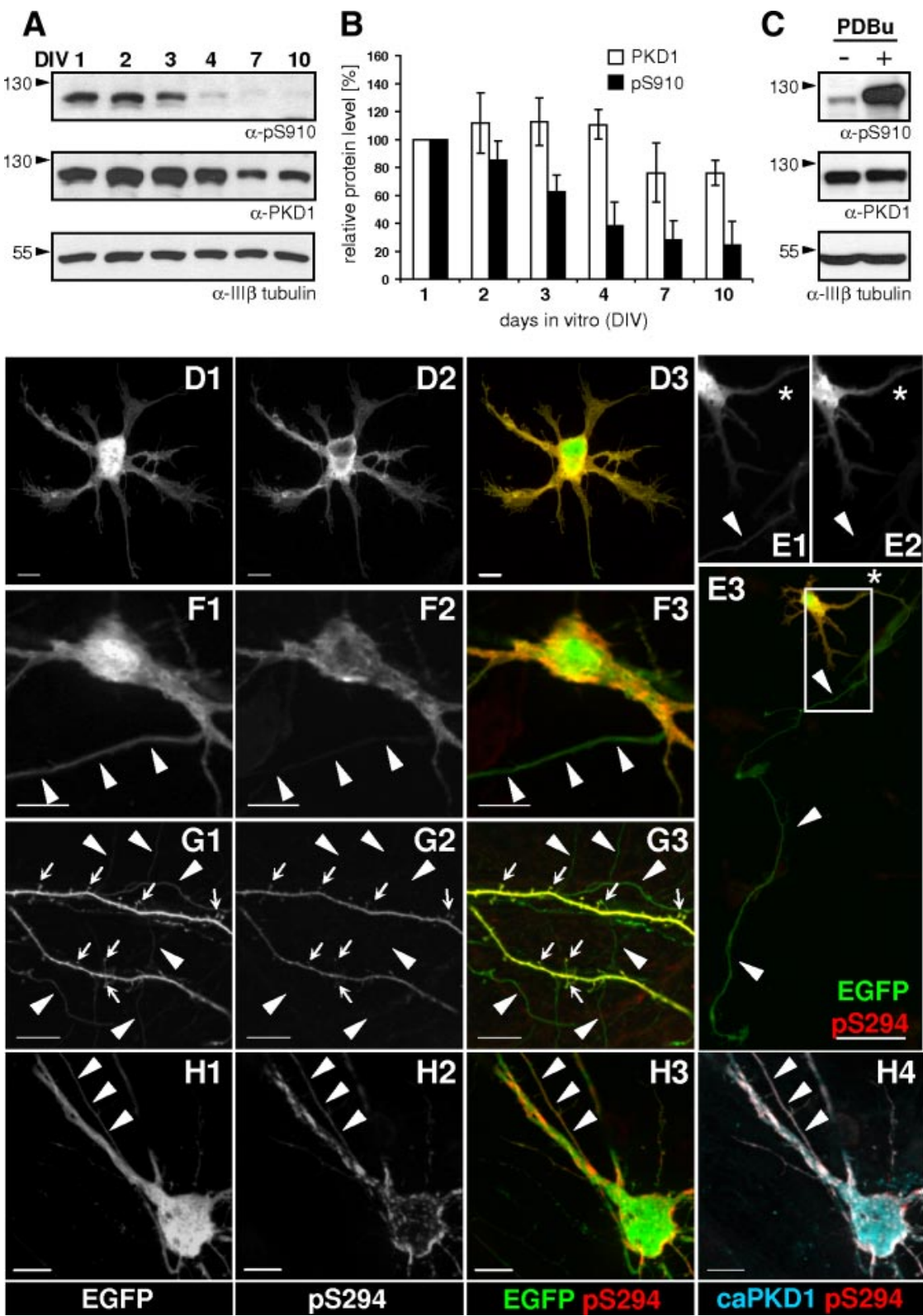

pS294
3

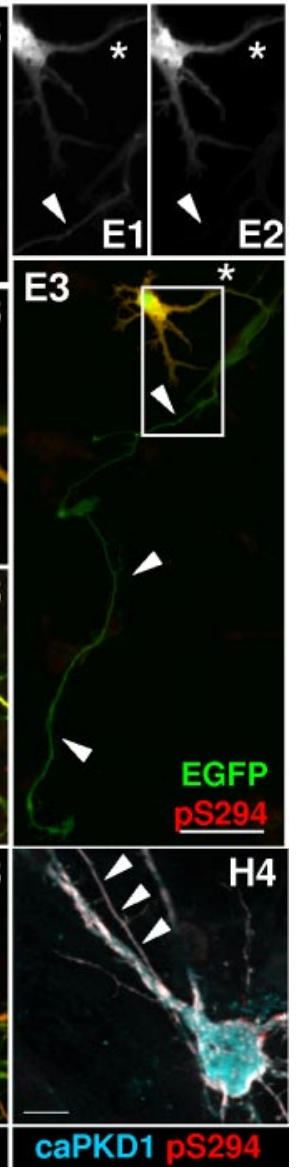

Figure 4. Endogenous PKD activity changes in parallel with neuronal maturation and is restricted to the mature somatodendritic compartment. (A) Endogenous PKD1 level as well as the extent of PKD1 autophosphorylation on S910 changed during the course of in vitro neuronal development. The pS910 blot was reprobed by the anti-PKD1 antibody. (B) Quantification of relative PKD1 expression levels. Relative total (empty bars) or autophosphorylated (black bars) PKD intensity values were correlated to the corresponding $\operatorname{III} \beta$ tubulin values, and then ratios were correlated to DIV1 values from the same gel. Graph shows the mean and SD calculated from four independent experiments. (C) Ten minutes of $1 \mu \mathrm{M}$ PDBu treatment highly increased endogenous PKD1 autophosphorylation in DIV10 neurons, whereas total PKD1 and III $\beta$-tubulin levels remained stable. (D) pS294 signal was uniformly detected in the soma and in the developing neurites of stage 2 hippocampal neurons. (E) Parallel to axonal development, PKDrep phosphorylation was excluded from the distal axon (indicated by arrowheads) and was restricted to the proximal axonal segment (asterisk) as well as to the soma and dendrites of a stage 3 neuron. E1 and E2 show the EGFP or pS294 signal, respectively, from the boxed area on E3. (F and G) In DIV10 neurons, PKDrep phosphorylation was absent from the initial axonal segment and thin axon collaterals (arrowheads) but was clearly detected in the soma, in the dendrites, and in the dendritic spines (small arrows). (H) When neurons were cotransfected with the constitutive active PKD1 ${ }^{\mathrm{S} 738 / 742 \mathrm{E}}$-mCherry mutant (caPKD1), nontargeted PKD reporter was also phosphorylated in the axon (arrowheads). Note the increased surface localization of pSer294 signal in the soma of the cotransfected neuron as well. Pictures are projection images of four to six adjacent z-stacks taken by the Apotome system. Bars, $50 \mu \mathrm{m}$ (E3) and $10 \mu \mathrm{m}(\mathrm{D}$ and F-H). differ between the vector transfected neurons and wtPKD1EGFP or caPKD1-EGFP expressing neurons (Figure 5D).

The extent of Golgi complex dispersal (data not shown) and the ratio of affected neurons were in good correlation with the expression level of kdPKD1-EGFP or with the posttransfection time (Supplemental Table 1). These data indicate that dominant-negative effects of kdPKD1-EGFP develop gradually and emphasize the importance of PKD activity in the maintenance of the normal Golgi apparatus structure in neurons.

\section{Doxycycline induced kdPKD1-EGFP Expression in Hippocampal Neurons Leads to Disruption of Golgi Morphology In Vivo}

To confirm our in vitro results, we generated mice expressing human kdPKD1-EGFP under the control of the tetracycline-responsive (TetO) hCMV promoter by standard transgenic techniques and pronucleus injection (kdPKD1-EGFP mice; Figure 6). Several transgenic lines were established from founder animals. The presence of the transgene in the founder mice as well as in the offsprings was verified both by Southern analysis and with genomic PCR (Figure 6B; data not shown). kdPKD1-EGFP mice were crossed to mice carrying rtTA2 under the control of CaMKII $\alpha$ promoter, known to provide a forebrain and hippocampus-specific expression pattern of the transactivator (Michalon et al., 2005). Neither single transgenic, nor double transgenic mice showed any signs of abnormal development or behavioral effects (data not shown).

Expression of kdPKD1-EGFP was induced in adult CaMKII $\alpha$ rtTA2 $\times$ kdPKD1-EGFP mice only upon administration of Dox (Figure 6, C and D). The appearance of kdPKD1-EGFP signal was already visible upon $5 \mathrm{~d}$ of Dox treatment (data not shown). By 4 wk of feeding the animals with Dox, kdPKD1-EGFP expression exceeded the level of endogenous PKD expression in the hippocampus (Figure 

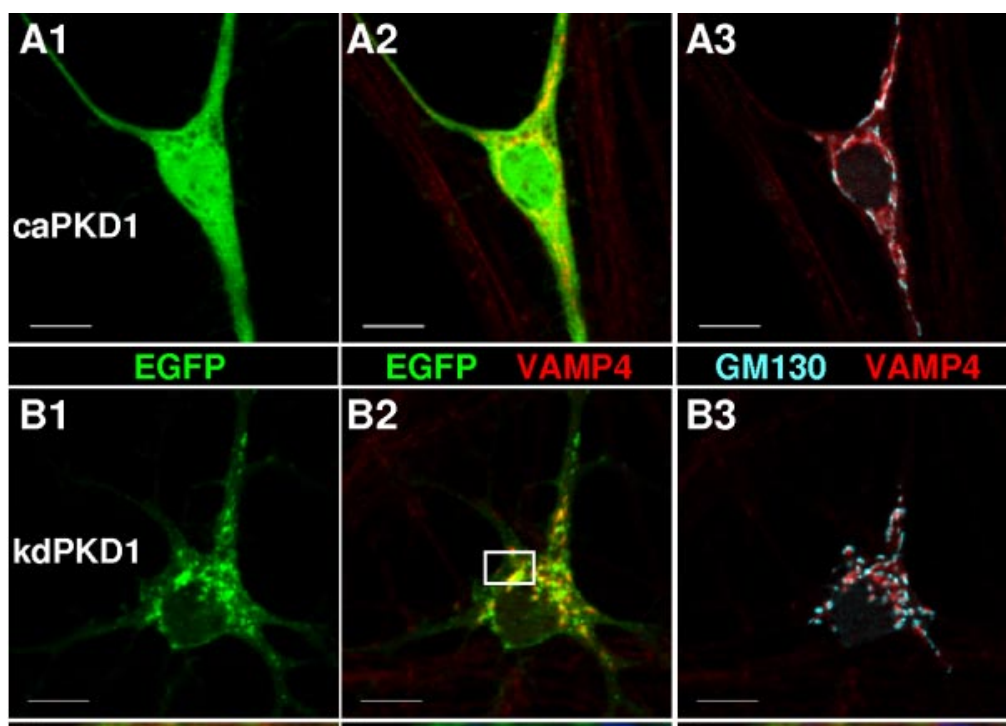

Figure 5. Golgi complex morphology in transfected hippocampal neurons. Hippocampal neurons were transfected with constitutive active (caPKD1-EGFP; A) or kinase inactive (kdPKD1-EGFP; $\mathrm{B}$ and C) mutant PKD1 constructs and were immunostained for VAMP4 and GM130 as trans- and cis-Golgi markers, respectively. (A) caPKD1-EGFP was distributed in the cytoplasm with clear enrichment at the Golgi structure and did not disturb thread-like neuronal Golgi structure. (B) kdPKD1-EGFP formed patches in the perinuclear region and in the dendrites and led to the dispersal of the Golgi complex. (C) Confocal images of the boxed area on B. kdPKD1-EGFP localized mainly to the VAMP4-positive, trans-Golgi side of the dispersed neuronal Golgi. Images are single $\mathrm{z}$-stacks recorded by the Apotome (A and $\mathrm{B}$ ) or the SP2 confocal (C) system. Bars, $10 \mu \mathrm{m}$ (A and B) or $2 \mu \mathrm{m}$ (C). See Supplemental Movie 2 and 3 for a 3D animation of the Golgi structure of the caPKD1EGFP-or kdPKD1-EGFP-transfected neurons, respectively. (D) Quantitative evaluation of neuronal Golgi morphology in 34 EGFP-, 46 wtPKD1-EGFP-, 37 kdPKD1-EGFP-, and 53 caPKD1-EGFP-transfected neurons. Data are shown as averages and SEM, ${ }^{*} \mathrm{p}<0.05$.
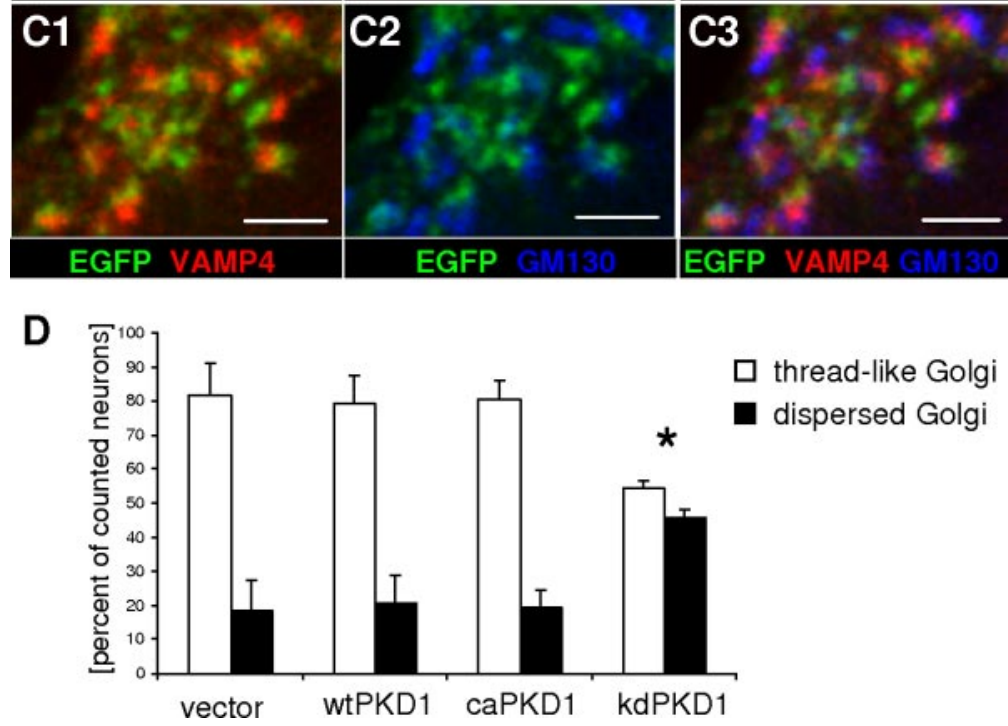

6D). On the second week of Dox treatment, transgene expression was evident in the perinuclear region and in the proximal dendrites of CA3 hippocampal neurons (Figure $6 \mathrm{E})$. As expected, no EGFP-expressing cells were found in wild-type animals (Figure 6F). Continuous Dox treatment for $4 \mathrm{wk}$ led to high level of transgene expression in the dentate gyrus (DG) and CA3, with somewhat lower signal in the CA1 region of double transgenic hippocampi (Figure 6G). Because of the partial activation of rtTA2 in the CaMKII $\alpha$ rtTA2 mice (Michalon et al., 2005), our observations revealed nonhomogenous expression patterns. However, most of the Dox-treated double transgenic hippocampal neurons were found to be kdPKD1-EGFP positive.

To visualize neuronal Golgi structure in vivo, VAMP4 immunostaining was used. Besides weakly labeling small, dot-like structures in the dendrites, resembling most likely recycling endosomes, VAMP4 staining was highly enriched at the neuronal Golgi complex (Figure 6, H and I). In nontransgenic hippocampal sections, regardless of the length of Dox treatment, VAMP4 was localized to thread-like, perinuclear structures often extending into the apical dendrites (Figure 6H). These results show that even long-term Dox treatment did not disturb normal, thread-like TGN morphology in wild-type neurons. In contrast, in CaMKII $\alpha$ rtTA2 $\times$

kdPKD1-EGFP double transgenic mice treated with Dox for 4 wk, kdPKD1-EGFP was enriched at VAMP4-positive TGN structures, which were strongly dispersed (Figure 6I). Thus, in vivo observations are in close agreement with the findings obtained from transfected hippocampal neuronal cultures.

\section{PKD Activity Regulates Dendritic Rearrangements in Neurons}

Our experiments have already shown that besides being active at the Golgi complex, endogenous PKD was selectively active in the dendrites (Figure 4). To investigate the effect of PKD1 mutants on dendritic arrangement, morphology of EGFP, wtPKD1-GFP, caPKD-GFP, or kdPKD1-GFPtransfected neurons was quantitatively analyzed in DIV10 hippocampal neurons, 22-24 h after transfection. Modified Sholl analysis was carried out to determine the extent of dendritic branching by calculating the intersection numbers at concentric circles located over the soma of the transfected neurons with a $20-\mu \mathrm{m}$ stepwise increase in radius (Figure 7A shows the outline and dendritic intersections of representative neurons). The average number of intersections as a function of distance from the soma characterizes well the dendritic arborization of the transfected neurons (Figure 7, B and $\mathrm{C}$ ). 

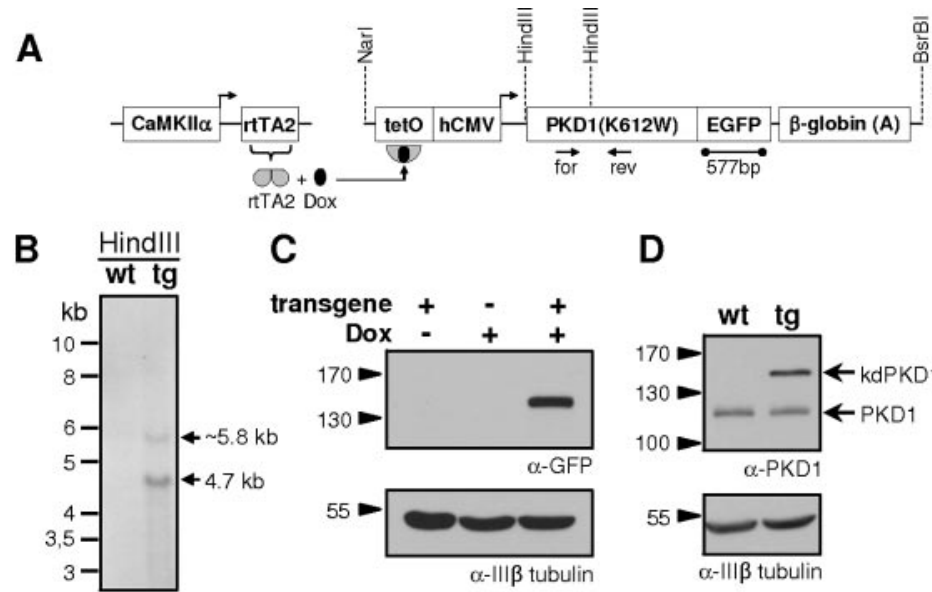

C
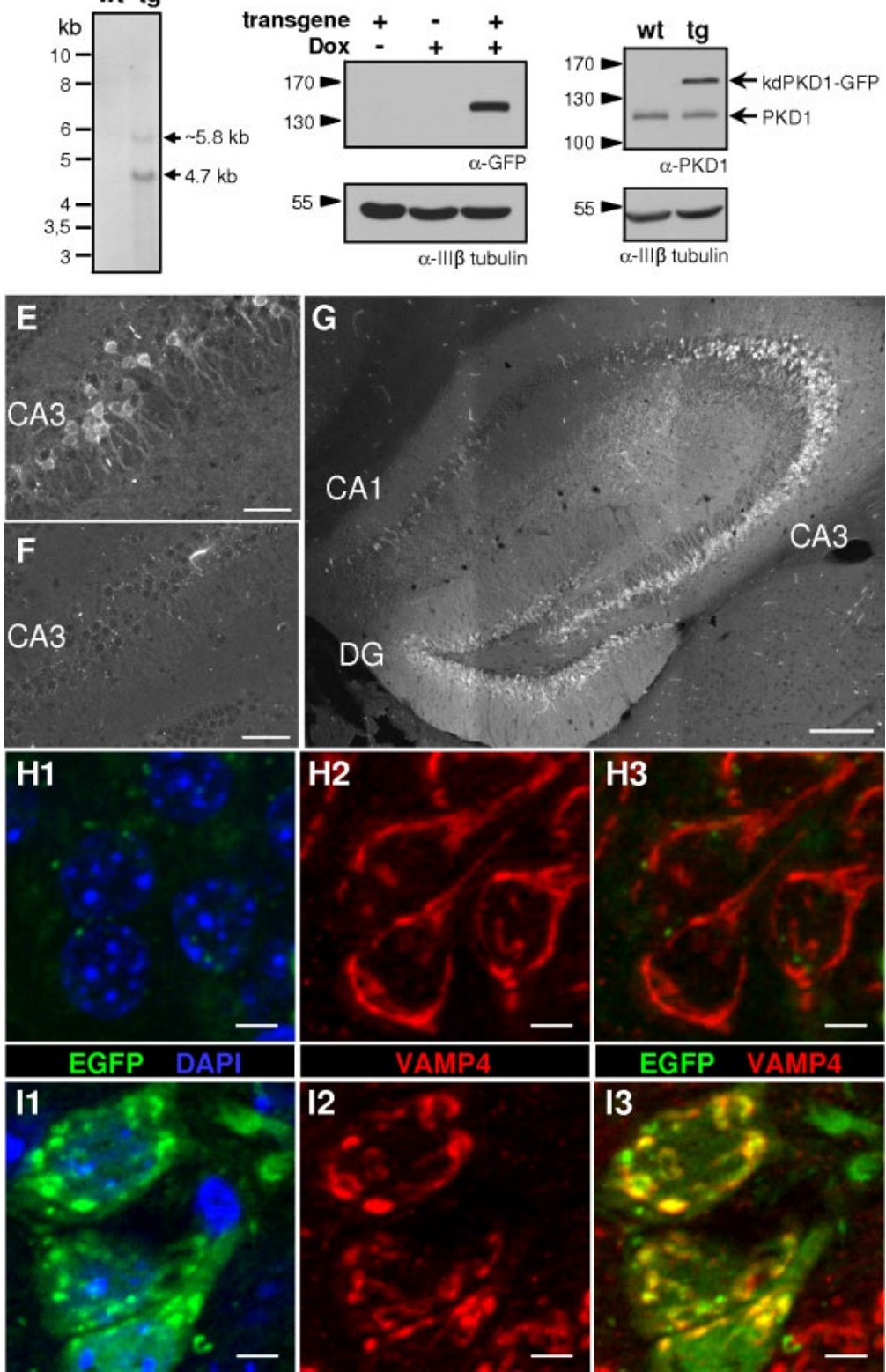

Figure 6. Characterization of in vivo kdPKD1-EGFP expression in CaMKII $\alpha$ rtTA2 $\times$ kdPKD1-EGFP transgenic mice. (A) Schematic diagram depicting the generation of CaMKII $\alpha$-rtTA2 $\times$ kdPKD1-EGFP double transgenic mice. Upon Dox treatment, rtTA2 binds to the tetO-responsive element and induces the expression of kdPKD1-EGFP. (B) Southern blot from wild-type (wt) and kdPKD1-EGFP transgenic ( $\mathrm{tg}$ ) mice, hybridized with the 577-bp EGFP probe indicted in A. (C) Transgene expression in hippocampal lysates was induced only upon Dox treatment of CaMKII $\alpha$ rtTA2 $\times$ kdPKD1-EGFP double transgenic (transgene) mice. (D) After 4 wk of Dox treatment, transgene expression level exceeded endogenous PKD1 level in hippocampal lysates from double transgenic mice. In wt samples, only endogenous PKD1 was detected. (E and F) In the CA3 region of double transgenic mice, kdPKD1-EGFP expression was already evident after 2 wk of Dox treatment (E), whereas sections from nontransgenic littermates showed no expression (F). (G) Four weeks of Dox treatment led to elevated hippocampal expression of kdPKD1-EGFP in double transgenic animals, especially in the DG and CA3 region of the hippocampus. In CA1, weaker expression was also detected. (H and I) CA3 neurons of Dox-treated (4 wk) wild-type $(\mathrm{H})$ and double transgenic (I) sections. Neuronal TGN was visualized by VAMP4 staining (H2-3, I2-3), nuclei were stained by DAPI. $G$ is a mosaic image from nine images taken by $10 \times$ objective and the Apotome system. E and F pictures are projection images of four to six adjacent $\mathrm{z}$-stacks obtained by a $20 \times$ objective, whereas $\mathrm{H}$ and I were recorded by a $63 \times$ objective and are the projections of 12-14 individual $z$-stacks. Corresponding pictures from wild-type and double transgenic sections were taken under identical settings and processed similarly. Bars, $200 \mu \mathrm{m}(\mathrm{G}), 50 \mu \mathrm{m}$ (E and $\mathrm{F})$, and $5 \mu \mathrm{m}$ ( $\mathrm{H}$ and $\mathrm{I})$.
Control, vector-only-transfected neurons had the highest number of intersections in the close (within $40 \mu \mathrm{m}$ ) vicinity of the soma, with dendrites reaching up to a $300-\mu \mathrm{m}$ distance from the soma. The extent of arborization gradually decreased in parallel with the increasing distance. The presence of kdPKD1-EGFP led to a significant decrease in the intersection numbers at almost all levels (Figure 7B, asterisks), and dendrites did not extend further than a $200-\mu \mathrm{m}$ distance. Introducing caPKD1-EGFP into transfected neurons, in contrast, led to a significant increase in dendritic arborization. Not only did the distribution of the highest intersection numbers shift toward outer regions around the soma $(60-80 \mu \mathrm{m})$ but also the average number of crosspoints was also significantly elevated (Figure 7B, + signs). Expression of wtPKD1-EGFP led to similar changes, but to a lesser extent.

To reveal potential correlation between dendritic arborization and Golgi complex morphology, high-magnification pictures were recorded from the soma region of EGFP and kdPKD1-EGFP-transfected neurons, followed by evaluating dendritic arborization of the same cells. In neurons transfected with EGFP, no correlation was found between the extent of dendritic arborization and Golgi complex morphology (average number of dendritic intersections 

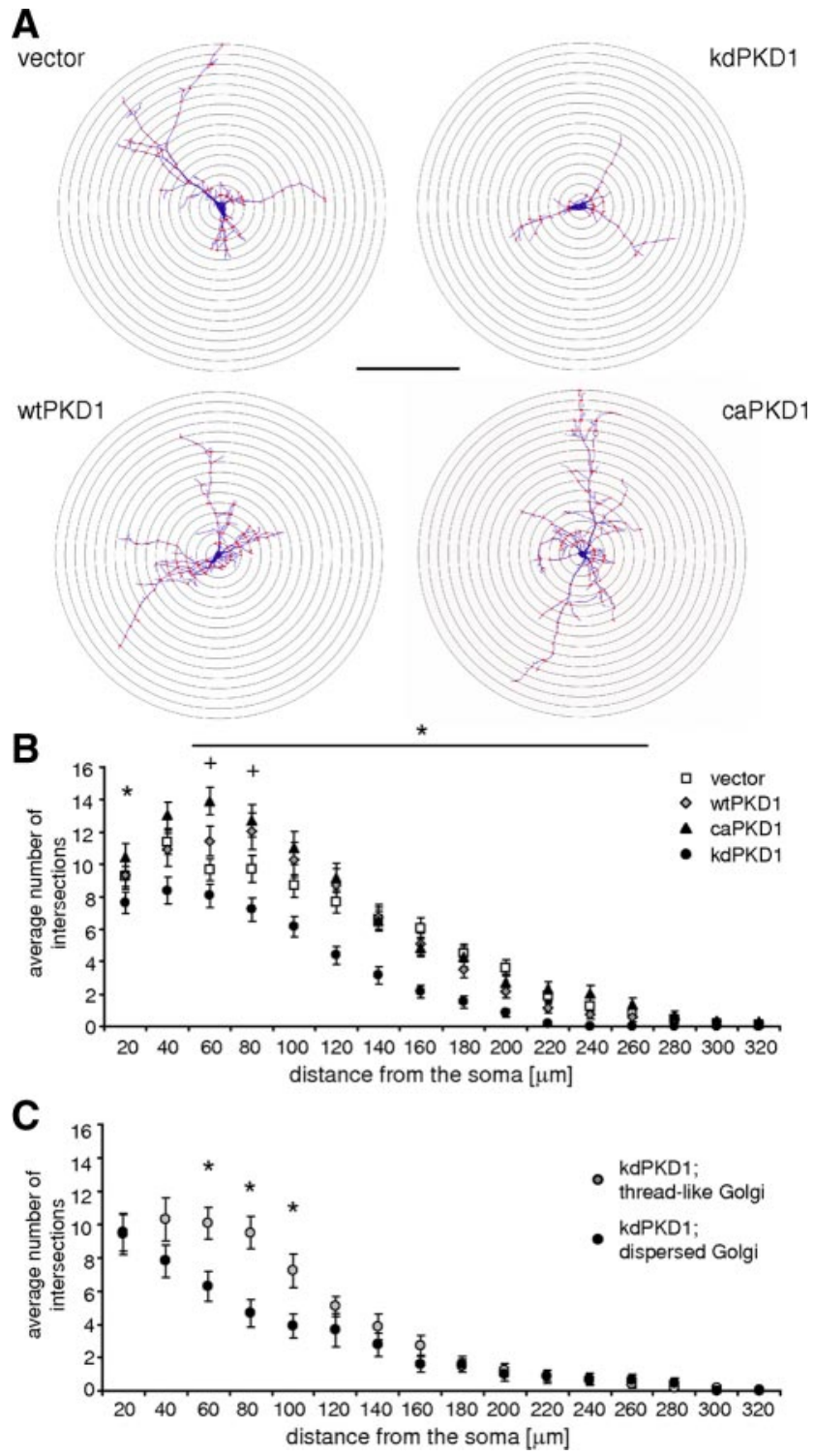

Figure 7. Sholl analysis of transfected hippocampal neurons. (A) Representative Sholl analysis images of neurons transfected with different constructs as indicated at the sides. Dendritic intersections are labeled by red dots. Bar indicates $200 \mu \mathrm{m}$. (B) Average intersection numbers at certain distance from the soma in neurons transfected with EGFP vector (empty squares), wtPKD1-EGFP (gray diamonds), caPKD1-EGFP (filled triangles), or kdPKD1-EGFP (filled circles). Twenty-seven EGFP-, 23 wtPKD1-EGFP-, 28 kdPKD1EGFP-, and 27 caPKD1-EGFP-transfected neurons were used for the analyses.(C) The extent of Golgi complex dispersal correlated with more reduced dendritic arborization in kdPKD1-transfected neurons. Thirty-seven kdPKD1-EGFP-transfected neurons were used for this analysis. Data are presented as averages and SEM, $\mathrm{p}<$ 0.05 for kdPKD1-EGFP- $(*)$ or for caPKD1-EGFP $(+)$-expressing neurons.

and SEM values were $76.45 \pm 6.92$ and $85.63 \pm 7.11$ for neurons having normal, thread-like or dispersed Golgi complex, respectively; 29 cells investigated). In kdPKD1-EGFPexpressing neurons, in contrast, strong correlation was found (Figure 7C; 37 cells investigated). Those neurons that possessed highly dispersed Golgi apparatus had an even more severely reduced dendritic arborization compared with those cells, where Golgi complex was at least partially preserved in a filamentous, thread-like state. Similar findings were observed already during shorter (12 and 18h) posttransfection times, when kdPKD1-EGFP-transfected neurons possessing dispersed Golgi apparatus had severely reduced dendritic tree, as well (data not shown). Importantly, the ratio of these cells increased with time (Supplemental Table 1).

\section{DISCUSSION}

Studies in polarized epithelial cells revealed that PKD is selectively involved in the basolateral membrane protein transport (Yeaman et al., 2004). In contrast, PKD-mediated effects and transport processes in neurons are still to be understood. In line with recent publications (Horton et al., 2005; Bisbal et al., 2008), our work provides additional evidences on the polarized, somatodendritic activity as well as on Golgi-localized action of PKD in neurons.

Our data clearly show that endogenous PKD activity is gradually restricted to the somatodendritic compartment of developing hippocampal neurons, leading to a highly polarized activity in mature, DIV10 cells. In neurons activated by diC8, a cell-permeable DAG analogue, or upon expression of caPKD1, pS294 immunostaining was highly increased at the plasma membrane in the soma and in the dendrites, reflecting local recruitment of activated PKD. Despite the presence of endogenous PKD1 in the axons (Supplemental Figure 2; Bisbal et al., 2008; Yin et al., 2008), treatment with diC8 did not increase axonal pS294 signal. In contrast, axonal PKD activity became detectable upon overexpression of exogenous PKDs, with caPKD1, wtPKD2, or wtPKD3 constructs all being evenly distributed throughout the neurons.

Initially, increased PKDrep phosphorylation upon wtPKD3 overexpression seems to be surprising because PI4KIII $\beta$ is known to be selectively phosphorylated on S294 by both PKD1 and PKD2 but not by PKD3 (Hausser et al., 2005). However, PKD isoforms have been shown to form dimeric complexes (Bossard et al., 2007), suggesting that PKD3 action on PKDrep phosphorylation was probably mediated via interaction with PKD1 and/or PKD2. Our PKD activity reporter constructs cannot be used to directly differentiate between PKD1- or PKD2-mediated processes. Nevertheless, based on PKD1 being expressed to a much higher extent in mature hippocampal neuronal cultures than PKD2 (data not shown; Bisbal et al., 2008), both Golgi-targeted and nontargeted PKD activity reporters indicated mostly the activation of endogenous PKD1. It is important to note that mutation in the ATP binding domain of PKD1 (K612W) was shown to exert a dominant-negative effect over cotransfected wtPKD1 and wtPKD2 as well. Therefore, kdPKD1 expression in neurons can inhibit endogenous PKD activity (regardless to the PKD isoforms) in a dominant manner.

As a feasible explanation to the lack of endogenous PKD activity in the axon, we can assume that inactivation of PKD, e.g., via sequestration by 14-3-3 adapter proteins (Hausser et al., 1999; Taya et al., 2007) is selectively increased in the axons. At the same time, selective dephosphorylation of PKD, the reporter, or both (Uetani et al., 2006) has to be also taken into consideration. Irrespective of the underlying mechanisms, our findings are in accordance with recent data showing that kdPKD1 expression had no axonal effects in DIV5 hippocampal neurons (Horton et al., 2005) or on the localization of axonal membrane proteins (Bisbal et al., 2008).

In young, nonpolarized neuronal cultures, even intracellular phosphorylation of PKDrep and high level of pS910 autophosphorylation was observed. The restriction of PKD activity to the somatodendritic compartments corresponded to the relative decrease in pS910 levels. In accordance with 
recent publications (Watkins et al., 2008; Yin et al., 2008), these results indicate that PKD is involved in early neuronal polarization processes. The multiple axon formation upon decreased PKD activity described by Yin et al. (2008), however, is in contrast to published data (Horton et al., 2005; Watkins et al., 2008) and also to our observations (Czöndör and Schlett, unpublished data). Thus, further work is needed to clarify these apparent inconsistencies.

Recently, impaired PKD activity in mature neurons was shown to induce increased endocytosis of dendritic membrane proteins (Bisbal et al., 2008), indicating that PKD activity is required for the selective maintenance of the dendritic membrane surface. In accordance with these findings, our results showed dramatic shrinkage of the dendritic tree upon kdPKD1 expression within $24 \mathrm{~h}$. In contrast, caPKD1 led to enhanced arborization in transfected neurons that can be a consequence of increased membrane surface due to inhibited dendritic endocytosis.

Besides endocytosis as a potential explanation for the observed changes in dendritic arborization, direct PKD action on Golgi-mediated secretory processes also has to be considered. Golgi-mediated secretory processes are known to play highly important role in the supply of cargo necessary for the maintenance of the dendritic surface machinery (Ye et al., 2007; Hanus and Ehlers, 2008; Tang, 2008). Therefore, impaired dendrite-directed secretory transport can also result in the shrinkage of the dendritic tree in kdPKD1transfected neurons.

The importance of the Golgi structure necessary for the high turnover of dendritic membrane surface is reflected by the presence of so-called dendritic outposts in the main dendrites (Sytnyk et al., 2002; Horton and Ehlers, 2003). In our hippocampal cultures, Golgi-targeted PKD reporter revealed high endogenous PKD activity at the neuronal Golgi complex. Moreover, PKD1 constructs showed enrichment at the TGN, especially in kdPKD1 expression. It is known that the K612W mutation in the kinase domain of PKD1 does neither interfere with substrate binding nor with DAG-dependent membrane recruitment of the kinase (Johannes et al., 1998; Hausser et al., 2002). However, detachment from membranes seems impaired, resulting in an accumulation at the TGN (Maeda et al., 2001; Bard and Malhotra, 2006). Accordingly, accumulation of kdPKD1-GFP at the VAMP4positive TGN structures was observed both in vitro, in transfected neurons, and in vivo, upon Dox-induced PKD mutant expression in CaMKII $\alpha$ rtTA2 $\times$ kdPKD1-EGFP double transgenic mice.

Importantly, kdPKD1 accumulation led to the disruption of the neuronal Golgi apparatus via the formation of smaller fragments, still possessing cis- and trans-Golgi elements. Prolonged or elevated kdPKD1 expression level led to increased ratio of cells with impaired Golgi complex morphology in the investigated cultures as well as in vivo. These data indicate that a certain level of kdPKD1 expression is needed to affect Golgi morphology, likely because of the development of the dominant-negative effect over endogenous PKD isoforms. Differences in the expression level of kdPKD1 and the time point of analyses can also explain the discrepancy between our results and that of Bisbal et al. (2008), who did not report morphological changes in the Golgi apparatus upon interference with neuronal PKD activity.

Fragmentation of the Golgi apparatus is at variance with the well-described tubulation of the TGN in kdPKD-expressing nonneuronal cells, which is widely accepted as a consequence of impaired vesicle fission (Bard and Malhotra, 2006; De Matteis and Luini, 2008). However, morphology of the Golgi complex in neuronal cells differs largely from those of the typical transformed cell lines of epithelial origin. Therefore, despite potential differences in phenotypical changes, our results presented here and in earlier studies carried out in nonneuronal cells share the findings that 1) PKD activity is required for the maintenance of the cell type-specific Golgi complex architecture and that 2) interfering with PKD activity rapidly changes Golgi complex integrity. Because the extent of Golgi apparatus disruption in kdPKD1-transfected neurons correlated well with the extent of impaired dendritic arborization, we assume that PKD activity regulates the maintenance of dendritic tree both by acting at the Golgi apparatus as well as by influencing local endocytotic processes in the dendrites.

In addition, PKD activity is involved in the regulation of cytoskeletal organization affecting cell migration and invasion in nonneuronal cells (Van Lint et al., 2002; Qiang et al., 2004). PKD was also reported to interact with F-actin and cortactin (Eiseler et al., 2007) and to participate in nocodazole-mediated disruption of the Golgi complex (Fuchs et al., 2009). As the morphology of the Golgi complex depends to a large extent on the integrity of the surrounding cytoskeletal elements (Bard and Malhotra, 2006; Egea et al., 2006; De Matteis and Luini, 2008), the observed disruption of the Golgi complex could be also a consequence of altered cytoskeletal organization upon kdPKD1 expression. Additionally, cytoskeletal rearrangements fundamentally influence the formation and maintenance of dendritic structures, including elongation and branching of dendrites or spine formation (Gauthier-Campbell et al., 2004; Kim et al., 2006; Hayashi et al., 2007; Tada et al., 2007; Zhang and Macara, 2008). Accordingly, direct or indirect cytoskeletal effects of PKD can also participate in the observed dendritic rearrangements in transfected neurons, especially when taking into account that high level of PKD activity was observed not only around the neuronal Golgi but also in the cytoplasm of dendrites and even in the dendritic spines.

Together, our investigations have provided evidence that PKD acts selectively in the somatodendritic compartment of polarized neurons and is needed for the integrity of Golgi apparatus and for the maintenance of dendritic arborization.

\section{ACKNOWLEDGMENTS}

We are grateful to Dr. A. Jeromin (Allen Institute for Brain Science, Seattle, WA) for providing constructs. We are indebted to Gert Sonntag (Carl Zeiss) for providing the Zeiss HS Cellobserver system for the initial investigations and for useful advice on the technical possibilities. K. C. and K. S. received the European Molecular Biology Organization short-term fellowships. This work was supported by the National Office for Research and Technology (Cell-Kom RET to K. S.), by OTKA 61784 (to K..S.), by Deutsche Forschungsgemeinschaft HA3557/2-1 and 4-1 (to A. H. and K. P.), and by University Stuttgart CSB-CMF intramural funds (to K. P.).

\section{REFERENCES}

Bard, F., and Malhotra, V. (2006). The formation of TGN-to-plasma-membrane transport carriers. Annu. Rev. Cell Dev. Biol. 22, 439-455.

Baron, U., Freundlieb, S., Gossen, M., and Bujard, H. (1995). Co-regulation of two gene activities by tetracycline via a bidirectional promoter. Nucleic Acids Res. 23, 3605-3606.

Bisbal, M., Conde, C., Donoso, M., Bollati, F., Sesma, J., Quiroga, S., Diaz Anel, A., Malhotra, V., Marzolo, M. P., and Caceres, A. (2008). Protein kinase D regulates trafficking of dendritic membrane proteins in developing neurons. J. Neurosci. 28, 9297-9308.

Bossard, C., Bresson, D., Polishchuk, R. S., and Malhotra, V. (2007). Dimeric PKD regulates membrane fission to form transport carriers at the TGN. J. Cell Biol. 179, 1123-1131.

Bresler, T., Shapira, M., Boeckers, T., Dresbach, T., Futter, M., Garner, C. C., Rosenblum, K., Gundelfinger, E. D., and Ziv, N. E. (2004). Postsynaptic 
density assembly is fundamentally different from presynaptic active zone assembly. J. Neurosci. 24, 1507-1520.

De Matteis, M. A., and Luini, A. (2008). Exiting the Golgi complex. Nat. Rev. Mol. Cell. Biol. 9, 273-284.

Diaz Anel, A. M., and Malhotra, V. (2005). PKCeta is required for beta1gamma2/beta3gamma2- and PKD-mediated transport to the cell surface and the organization of the Golgi apparatus. J. Cell Biol. 169, 83-91.

Doppler, H., Storz, P., Li, J., Comb, M. J., and Toker, A. (2005). A phosphorylation state-specific antibody recognizes $\mathrm{Hsp} 27$, a novel substrate of protein kinase D. J. Biol. Chem. 280, 15013-15019.

Dresbach, T., Qualmann, B., Kessels, M. M., Garner, C. C., and Gundelfinger, E. D. (2001). The presynaptic cytomatrix of brain synapses. Cell. Mol. Life Sci. $58,94-116$.

Egea, G., Lazaro-Dieguez, F., and Vilella, M. (2006). Actin dynamics at the Golgi complex in mammalian cells. Curr. Opin. Cell Biol. 18, 168-178.

Eiseler, T., Schmid, M. A., Topbas, F., Pfizenmaier, K., and Hausser, A. (2007). PKD is recruited to sites of actin remodelling at the leading edge and negatively regulates cell migration. FEBS Lett. 581, 4279-4287.

Ellwanger, K., Pfizenmaier, K., Lutz, S., and Hausser, A. (2008). Expression patterns of protein kinase D 3 during mouse development. BMC Dev. Biol. 8, 47.

Fuchs, Y. F., Eisler, S. A., Link, G., Schlicker, O., Bunt, G., Pfizenmaier, K., and Hausser, A. (2009). A Golgi PKD activity reporter reveals a crucial role of PKD in nocodazole-induced Golgi dispersal. Traffic (in press).

Fugmann, T., Hausser, A., Schoffler, P., Schmid, S., Pfizenmaier, K., and Olayioye, M. A. (2007). Regulation of secretory transport by protein kinase D-mediated phosphorylation of the ceramide transfer protein. J. Cell Biol. 178, $15-22$.

Fujita, Y., and Okamoto, K. (2005). Golgi apparatus of the motor neurons in patients with amyotrophic lateral sclerosis and in mice models of amyotrophic lateral sclerosis. Neuropathology 25, 388-394.

Gardiol, A., Racca, C., and Triller, A. (1999). Dendritic and postsynaptic protein synthetic machinery. J. Neurosci. 19, 168-179.

Gauthier-Campbell, C., Bredt, D. S., Murphy, T. H., and El-Husseini Ael, D. (2004). Regulation of dendritic branching and filopodia formation in hippocampal neurons by specific acylated protein motifs. Mol. Biol. Cell 15, 2205-2217.

Ghanekar, Y., and Lowe, M. (2005). Protein kinase D: activation for Golgi carrier formation. Trends Cell Biol. 15, 511-514.

Hanus, C., and Ehlers, M. D. (2008). Secretory outposts for the local processing of membrane cargo in neuronal dendrites. Traffic 9, 1437-1445.

Hausser, A., Link, G., Bamberg, L., Burzlaff, A., Lutz, S., Pfizenmaier, K., and Johannes, F. J. (2002). Structural requirements for localization and activation of protein kinase C mu (PKC mu) at the Golgi compartment. J. Cell Biol. 156, 65-74.

Hausser, A., Storz, P., Link, G., Stoll, H., Liu, Y. C., Altman, A., Pfizenmaier, K., and Johannes, F. J. (1999). Protein kinase C mu is negatively regulated by 14-3-3 signal transduction proteins. J. Biol. Chem. 274, 9258-9264.

Hausser, A., Storz, P., Martens, S., Link, G., Toker, A., and Pfizenmaier, K. (2005). Protein kinase D regulates vesicular transport by phosphorylating and activating phosphatidylinositol-4 kinase IIIbeta at the Golgi complex. Nat. Cell Biol. 7, 880-886.

Haworth, R. S., and Avkiran, M. (2001). Inhibition of protein kinase D by resveratrol. Biochem. Pharmacol. 62, 1647-1651.

Hayashi, K., Ohshima, T., Hashimoto, M., and Mikoshiba, K. (2007). Pak1 regulates dendritic branching and spine formation. Dev. Neurobiol. 67, 655669

Horton, A. C., and Ehlers, M. D. (2003). Dual modes of endoplasmic reticulum-to-Golgi transport in dendrites revealed by live-cell imaging. J. Neurosci. 23, 6188-6199.

Horton, A. C., and Ehlers, M. D. (2004). Secretory trafficking in neuronal dendrites. Nat. Cell Biol. 6, 585-591.

Horton, A. C., Racz, B., Monson, E. E., Lin, A. L., Weinberg, R. J., and Ehlers, M. D. (2005). Polarized secretory trafficking directs cargo for asymmetric dendrite growth and morphogenesis. Neuron 48, 757-771.

Johannes, F. J., Horn, J., Link, G., Haas, E., Siemienski, K., Wajant, H., and Pfizenmaier, K. (1998). Protein kinase Cmu downregulation of tumor-necrosis-factor-induced apoptosis correlates with enhanced expression of nuclearfactor-kappaB-dependent protective genes. Eur. J. Biochem. 257, 47-54.

Johannes, F. J., Prestle, J., Dieterich, S., Oberhagemann, P., Link, G., and Pfizenmaier, K. (1995). Characterization of activators and inhibitors of protein kinase C mu. Eur. J. Biochem. 227, 303-307.
Kim, Y., et al. (2006). Phosphorylation of WAVE1 regulates actin polymerization and dendritic spine morphology. Nature 442, 814-817.

Lein, P. J., Guo, X., Shi, G. X., Moholt-Siebert, M., Bruun, D., and Andres, D. A (2007). The novel GTPase Rit differentially regulates axonal and dendritic growth. J. Neurosci. 27, 4725-4736.

Liljedahl, M., Maeda, Y., Colanzi, A., Ayala, I., Van Lint, J., and Malhotra, V. (2001). Protein kinase D regulates the fission of cell surface destined transport carriers from the trans-Golgi network. Cell 104, 409-420.

Maeda, Y., Beznoussenko, G. V., Van Lint, J., Mironov, A. A., and Malhotra, V. (2001). Recruitment of protein kinase D to the trans-Golgi network via the first cysteine-rich domain. EMBO J. 20, 5982-5990.

Matthews, S., Iglesias, T., Cantrell, D., and Rozengurt, E. (1999). Dynamic re-distribution of protein kinase D (PKD) as revealed by a GFP-PKD fusion protein: dissociation from PKD activation. FEBS Lett. 457, 515-521.

Michalon, A., Koshibu, K., Baumgartel, K., Spirig, D. H., and Mansuy, I. M. (2005). Inducible and neuron-specific gene expression in the adult mouse brain with the rtTA2S-M2 system. Genesis 43, 205-212.

Nagy, A. (2003). Manipulating the Mouse Embryo: A Laboratory Manual, Cold Spring Harbor, NY: Cold Spring Harbor Laboratory Press.

Nakamura, N., Rabouille, C., Watson, R., Nilsson, T., Hui, N., Slusarewicz, P., Kreis, T. E., and Warren, G. (1995). Characterization of a cis-Golgi matrix protein, GM130. J. Cell Biol. 131, 1715-1726.

Nishikawa, K., Toker, A., Johannes, F. J., Songyang, Z., and Cantley, L. C. (1997). Determination of the specific substrate sequence motifs of protein kinase C isozymes. J. Biol. Chem. 272, 952-960.

Oster, H., Abraham, D., and Leitges, M. (2006). Expression of the protein kinase D (PKD) family during mouse embryogenesis. Gene Expr. Patterns 6 , 400-408.

Pierce, J. P., Mayer, T., and McCarthy, J. B. (2001). Evidence for a satellite secretory pathway in neuronal dendritic spines. Curr. Biol. 11, 351-355.

Qiang, Y. W., Yao, L., Tosato, G., and Rudikoff, S. (2004). Insulin-like growth factor I induces migration and invasion of human multiple myeloma cells. Blood 103, 301-308.

Rey, O., Reeve, J. R., Jr., Zhukova, E., Sinnett-Smith, J., and Rozengurt, E. (2004). G protein-coupled receptor-mediated phosphorylation of the activation loop of protein kinase $\mathrm{D}$ : dependence on plasma membrane translocation and protein kinase Cepsilon. J. Biol. Chem. 279, 34361-34372.

Rykx, A., De Kimpe, L., Mikhalap, S., Vantus, T., Seufferlein, T., Vandenheede, J. R., and Van Lint, J. (2003). Protein kinase D: a family affair. FEBS Lett. 546, $81-86$.

Sheng, M. (2001). Molecular organization of the postsynaptic specialization. Proc. Natl. Acad. Sci. USA 98, 7058-7061.

Silverman, M. A., Kaech, S., Jareb, M., Burack, M. A., Vogt, L., Sonderegger P., and Banker, G. (2001). Sorting and directed transport of membrane proteins during development of hippocampal neurons in culture. Proc. Natl. Acad. Sci. USA 98, 7051-7057.

Steegmaier, M., Klumperman, J., Foletti, D. L., Yoo, J. S., and Scheller, R. H. (1999). Vesicle-associated membrane protein 4 is implicated in trans-Golgi network vesicle trafficking. Mol. Biol. Cell 10, 1957-1972.

Sytnyk, V., Leshchyns'ka, I., Delling, M., Dityateva, G., Dityatev, A., and Schachner, M. (2002). Neural cell adhesion molecule promotes accumulation of TGN organelles at sites of neuron-to-neuron contacts. J. Cell Biol. 159, 649-661.

Tada, T., Simonetta, A., Batterton, M., Kinoshita, M., Edbauer, D., and Sheng, M. (2007). Role of Septin cytoskeleton in spine morphogenesis and dendrite development in neurons. Curr. Biol. 17, 1752-1758.

Takamine, K., Okamoto, K., Fujita, Y., Sakurai, A., Takatama, M., and Gonatas, N. K. (2000). The involvement of the neuronal Golgi apparatus and trans-Golgi network in the human olivary hypertrophy. J. Neurol. Sci. 182, 45-50.

Takemoto-Kimura, S., Ageta-Ishihara, N., Nonaka, M., Adachi-Morishima, A., Mano, T., Okamura, M., Fujii, H., Fuse, T., Hoshino, M., Suzuki, S., Kojima, M., Mishina, M., Okuno, H., and Bito, H. (2007). Regulation of dendritogenesis via a lipid-raft-associated $\mathrm{Ca} 2+/$ calmodulin-dependent protein kinase CLICK-III/CaMKIgamma. Neuron 54, 755-770.

Tang, B. L. (2008). Emerging aspects of membrane traffic in neuronal dendrite growth. Biochim. Biophys. Acta 1783, 169-176.

Taya, S., Shinoda, T., Tsuboi, D., Asaki, J., Nagai, K., Hikita, T., Kuroda, S., Kuroda, K., Shimizu, M., Hirotsune, S., Iwamatsu, A., and Kaibuchi, K. (2007). DISC1 regulates the transport of the NUDEL/LIS1/14-3-3epsilon complex through kinesin-1. J. Neurosci. 27, 15-26. 
Tran, T. H., Zeng, Q., and Hong, W. (2007). VAMP4 cycles from the cell surface to the trans-Golgi network via sorting and recycling endosomes. J. Cell Sci. 120, 1028-1041.

Uetani, N., Chagnon, M. J., Kennedy, T. E., Iwakura, Y., and Tremblay, M. L. (2006). Mammalian motoneuron axon targeting requires receptor protein tyrosine phosphatases sigma and delta. J. Neurosci. 26, 5872-5880.

Van Lint, J., Rykx, A., Maeda, Y., Vantus, T., Sturany, S., Malhotra, V., Vandenheede, J. R., and Seufferlein, T. (2002). Protein kinase D: an intracellular traffic regulator on the move. Trends Cell Biol. 12, 193-200.

Wang, Q. J. (2006). PKD at the crossroads of DAG and PKC signaling. Trends Pharmacol. Sci. 27, 317-323.

Watkins, J. L., Lewandowski, K. T., Meek, S. E., Storz, P., Toker, A., and Piwnica-Worms, H. (2008). Phosphorylation of the Par-1 polarity kinase by protein kinase D regulates $14-3-3$ binding and membrane association. Proc. Natl. Acad. Sci. USA 105, 18378-18383.

Winckler, B., and Mellman, I. (1999). Neuronal polarity: controlling the sorting and diffusion of membrane components. Neuron 23, 637-640.
Ye, B., Zhang, Y., Song, W., Younger, S. H., Jan, L. Y., and Jan, Y. N. (2007) Growing dendrites and axons differ in their reliance on the secretory pathway. Cell 130, 717-729.

Yeaman, C., Ayala, M. I., Wright, J. R., Bard, F., Bossard, C., Ang, A., Maeda Y., Seufferlein, T., Mellman, I., Nelson, W. J., and Malhotra, V. (2004). Protein kinase $\mathrm{D}$ regulates basolateral membrane protein exit from trans-Golgi network. Nat. Cell Biol. 6, 106-112.

Yin, D. M., Huang, Y. H., Zhu, Y. B., and Wang, Y. (2008). Both the establishment and maintenance of neuronal polarity require the activity of protein kinase D in the Golgi apparatus. J. Neurosci. 28, 8832-8843.

Zhang, H., and Macara, I. G. (2008). The PAR-6 polarity protein regulates dendritic spine morphogenesis through p190 RhoGAP and the Rho GTPase. Dev. Cell 14, 216-226.

Zugaza, J. L., Sinnett-Smith, J., Van Lint, J., and Rozengurt, E. (1996). Protein kinase D (PKD) activation in intact cells through a protein kinase C-dependent signal transduction pathway. EMBO J. 15, 6220-6230. 